\title{
Fractional Quantum Field Theory: From Lattice to Continuum
}

\author{
Vasily E. Tarasov \\ Skobeltsyn Institute of Nuclear Physics, Lomonosov Moscow State University, Moscow 119991, Russia \\ Correspondence should be addressed to Vasily E. Tarasov; tarasov@theory.sinp.msu.ru
}

Received 23 August 2014; Accepted 8 October 2014; Published 30 October 2014

Academic Editor: Elias C. Vagenas

Copyright (C) 2014 Vasily E. Tarasov. This is an open access article distributed under the Creative Commons Attribution License, which permits unrestricted use, distribution, and reproduction in any medium, provided the original work is properly cited. The publication of this article was funded by SCOAP ${ }^{3}$.

An approach to formulate fractional field theories on unbounded lattice space-time is suggested. A fractional-order analog of the lattice quantum field theories is considered. Lattice analogs of the fractional-order 4-dimensional differential operators are proposed. We prove that continuum limit of the suggested lattice field theory gives a fractional field theory for the continuum 4-dimensional space-time. The fractional field equations, which are derived from equations for lattice space-time with long-range properties of power-law type, contain the Riesz type derivatives on noninteger orders with respect to space-time coordinates.

\section{Introduction}

Fractional calculus and fractional differential equations $[1$, 2] have a wide application in mechanics and physics. The theory of integrodifferential equations of noninteger orders is powerful tool to describe the dynamics of systems and processes with power-law nonlocality, long-range memory, and/or fractal properties. Recently the spatial fractionalorder derivatives have been actively used in the spacefractional quantum mechanics suggested in $[3,4]$, the quantization of fractional derivatives [5], the fractional Heisenberg and quantum Markovian equations [6,7], the fractional theory of open quantum systems $[8,9]$, the quantum field theory and gravity for fractional space-time $[10,11]$, and the fractional quantum field theory at positive temperature $[12,13]$. Fractional calculus allows us to take into account fractional power-law nonlocality of continuously distributed systems. Using the fractional calculus, we can consider space-time fractional differential equations in the quantum field theory. The fractional-order Laplace and d'Alembert operators have been suggested by Riesz in [14] in 1949 for the first time. Then noninteger powers of d'Alembertian are considered in different works (for example, see Section 28 in [1] and [1517]). The fractional Laplace and d'Alembert operators of the by Riesz type are a base in the construction of the fractional field theory in multidimensional spaces. As it was shown in [18-20], the continuum equations with fractional derivatives of the Riesz type can be directly connected to lattice models with long-range properties. A connection between the dynamics of lattice system with long-range properties and the fractional continuum equations is proved by using the transform operation [18-20] and it has been applied for the media with spatial dispersion law $[21,22]$, for the fields in fractional nonlocal materials [23,24], for fractional statistical mechanics [25], and for nonlinear classical fields [26].

A characteristic feature of continuum quantum field theories, which are used in high-energy physics, is ultraviolet divergences. The divergences arise in momentum (wavevector) space from modes of very high wave number, that is, the structure of the field theories at very short distances. In the narrow class of quantum theories, which are called "renormalizable," the divergences can be removed by a singular redefinition of the parameters of the theory. This process is called the renormalization [27], and it defines a quantum field theory as a nontrivial limit of theory with an ultraviolet cut-off. The renormalization requires the regularization of the path integrals in momentum space. These regularized integrals depend on parameters such as the momentum cutoff, the Pauli-Villars masses, and the dimensional regularization parameter, which are used in the correspondent regularization procedure. This regularized integration is ultraviolet finite. In some sense, we can say that the regularization procedure consists in the introduction of a momentum cut-off. 
In quantum field theory, the path integral approach is very important to describe processes in high energy physics [28]. The path integrals are well-defined for systems with a denumerable number of degrees of freedom. In field theory, we are dealing with the case of an innumerable number of degrees of freedom, labeled by the space-time coordinates at least. To give the path integrals a precise meaning, we can discretize space and time; that is, we can introduce a space-time lattice. The introduction of a lattice space-time corresponds to a special form of regularization of the path integrals. In the lattice field theory, the momentum space integrals will be cut off at a momentum of the order of the inverse lattice constants. The lattice regularization can be considered as a natural introduction of a momentum cut-off. The lattice renormalization procedure can be carried out for path integrals in momentum space. The first step of the procedure is regularization that consists in introducing a space-time lattice. This regularization allows us to give an exact definition of the path integral since the lattice has the denumerable number of degrees of freedom. Moreover the existence of the momentum cut-off is not surprising in the lattice field theories. In the expression of the Fourier integral for lattice fields, the momentum integration with respect to wave-vector components $k_{\mu}(\mu=1,2,3,4)$ is restricted by the Brillouin zone $k_{\mu} \in\left[-\pi / a_{\mu}, \pi / a_{\mu}\right]$, where $a_{\mu}$ are the lattice constants. The second step of the renormalization is a continualization procedure that removes the lattice structure by a continuum limit, where the lattice constants $a_{\mu}$ tend to zero. In this step of the renormalization process the momentum cut-off is removed by the continuum limit.

At the present time, fractional-order generalization of the lattice field theories has not been suggested. Lattice approach to the fractional field theories was not previously considered. In this paper, we propose a formulation of fractional field theory on a lattice space-time. The suggested theory can be considered as a lattice analog of the fractional field theories, and as a fractional-order analog of the lattice quantum field theories. For simplification, we consider the free scalar fields. It allows us to demonstrate a number of important properties in details. The lattice analogs of the fractional-order differential operators are suggested. We prove that continuum limit of the suggested lattice theory gives the fractional field theory with continuum space-time. The fractional field equations contain the Riesz type derivatives on noninteger orders with respect to space-time coordinates. In Section 2, the fractional field theory on continuum space-time is considered for scalar fields and fractional-order differential operators are defined.

In Section 3, the fractional-order lattice differential operators of noninteger orders are considered and the lattice fractional field theory for lattice space-time is proposed. In Section 4, the lattice-continuum transformation of the lattice fractional theories is discussed. A short conclusion is given in Section 5 .

\section{Fractional Field Theory on Continuum Space-Time}

2.1. Scalar Field in Pseudo-Euclidean Space-Time. For simplification, we consider scalar fields in the 4-dimensional
pseudo-Euclidean space-time $\mathbb{R}_{1,3}^{4}$. Let us consider the classical field equation

$$
\left(\square+M^{2}\right) \varphi(x)=J(x),
$$

where $\square$ is the d'Alembert operator, $\varphi(x)$ is a real field, and $x \in \mathbb{R}_{1,3}^{4}$ is the space-time vector with components $x_{\mu}$, where $\mu=0,1,2,3$. This field equation follows from stationary action principle, $\delta S[\varphi]=0$, where the action $S[\varphi]$ has the form

$$
S[\varphi]=-\frac{1}{2} \int d^{4} x \varphi(x)\left(\square+M^{2}\right) \varphi(x) .
$$

In the quantum theory the generalized coordinates $\varphi(x)$ and momenta $\dot{\varphi}(x)$ become the operators, $\Phi(x)$ and $\dot{\Phi}(x)$, that satisfy the canonical commutation relations. The Green functions are

$$
G_{s}\left(x_{1}, \ldots, x_{s}\right)=\left\langle\Omega\left|T\left\{\Phi\left(x_{1}\right) \cdots \Phi\left(x_{s}\right)\right\}\right| \Omega\right\rangle,
$$

where $|\Omega\rangle$ denotes the ground state "physical vacuum" of the fields and $T\{\}$ denotes the time-ordered product of the operators $\Phi(x)$. These Green functions have a path integral representation [28] in the form

$$
G_{s}\left(x_{1}, \ldots, x_{s}\right)=\frac{\int D \varphi\left(\varphi\left(x_{1}\right) \cdots \varphi\left(x_{s}\right)\right) e^{i S[\varphi]}}{\int D \varphi e^{i S[\varphi]}}
$$

where $\int D \varphi$ is the sum over all possible configurations of the field $\varphi(x)$. The effects arising from quantum fluctuations are defined by those contributions to the integral (4) that come from field configurations which are not solutions of the classical field equation (1) and hence do not lead to a stationary action.

2.2. From Pseudo-Euclidean to Euclidean Space-Time. Let us consider the analytic continuation of (4) to imaginary times (see Section 7.4 in [29]) such that

$$
x^{0} \longrightarrow-i x_{4}
$$

We will use $x_{E}$ to denote the Euclidean four-vector. It allows us to consider the Euclidean space-time $\mathbb{R}^{4}$ instead of the pseudo-Euclidean space-time $\mathbb{R}_{13}^{4}$.

The Euclidean action $S_{E}\left[\varphi_{c}\right]$ is obtained from the action (2) by using the three steps:

(1) firstly, the replacement $x^{0} \rightarrow-i x_{4}$, where $x^{0}$ appears explicitly,

(2) the use of the real valued field $\varphi_{c}\left(x_{E}\right)=\varphi_{c}\left(\vec{x}, x_{4}\right)$ instead of $\varphi(\vec{x}, t)$, where $\varphi_{c}\left(\vec{x}, x_{4}\right)$ is not obtained from $\varphi(t, \vec{x})$ by substituting $x_{4}$ for $t$, noting that $\varphi_{c}\left(x_{E}\right)=$ $\varphi_{c}\left(\vec{x}, x_{4}\right)$ is a real field which is a function of the Euclidean variable $x_{E}$,

(3) multiplying the resultant expression by $-i$.

As a result, this leads to the expression

$$
S_{E}\left[\varphi_{c}\right]=\frac{1}{2} \int d^{4} x_{E} \varphi_{c}\left(x_{E}\right)\left(-\square_{E}+M^{2}\right) \varphi_{c}\left(x_{E}\right),
$$


where $\square_{E}$ denotes the 4-dimensional Laplacian

$$
\square_{E}=\sum_{\mu=1}^{4} \frac{\partial^{2}}{\partial x_{E \mu}^{2}} .
$$

To formulate a fractional generalization of the field theory, we should use the physically dimensionless space-time coordinates. It allows us to have the same physical dimensions for all other physical values as in the usual (nonfractional) field theories with dimensionless coordinates. In this paper all the quantities will be done physically dimensionless to simplify our consideration. For this aim, we scale the mass parameter $M$, the coordinates $x_{E}$, and the field $\varphi_{c}$ according to their physical dimension. As seen from (6) the quantities $\varphi_{c}$ and $M$ have the physical dimension that is the inverse length, and it is obviously $x_{E}$ that has the dimension of length, since the action is dimensionless. Therefore we can define the dimensionless quantities $M_{C}, \mathbf{x}$, and $\varphi_{C}$ by the replacement:

$$
\begin{gathered}
\mathbf{x}=l_{0}^{-1} x_{E}, \quad x_{\mu}=l_{0}^{-1} x_{E \mu}, \quad \varphi_{C}=l_{0}^{-1} \varphi_{c}, \\
M_{C}=l_{0}^{-1} M .
\end{gathered}
$$

We will use $\mathbf{x}$ (instead of $x_{E}$ ) to denote the Euclidean fourvector with components $x_{\mu}$, where $\mu=1,2,3,4$. As a result, this leads to the expression for the Euclidean action $S_{E}\left[\varphi_{C}\right]$ is given by the equation

$$
S_{E}\left[\varphi_{C}\right]=\frac{1}{2} \int d^{4} \mathbf{x} \varphi_{C}(\mathbf{x})\left(-\square_{E, C}+M_{C}^{2}\right) \varphi_{C}(\mathbf{x}),
$$

where $\square_{E, C}$ denotes the 4-dimensional Laplacian for dimensionless variables $\mathbf{x}$ of continuum space-time, such that

$$
\square_{E, C}=\sum_{\mu=1}^{4} \frac{\partial^{2}}{\partial x_{\mu}^{2}}
$$

The Green functions (3), which are continued to imaginary times, have the path integral representation

$$
\left\langle\varphi_{C}\left(\mathbf{x}_{1}\right) \cdots \varphi_{C}\left(\mathbf{x}_{s}\right)\right\rangle_{E}=\frac{\int D \varphi_{C}\left(\varphi_{C}\left(\mathbf{x}_{1}\right) \cdots \varphi_{C}\left(\mathbf{x}_{s}\right)\right) e^{-S_{E}\left[\varphi_{C}\right]}}{\int D \varphi e^{-S_{E}\left[\varphi_{C}\right]}}
$$

where we use the notation for the Euclidean Green function for physically dimensionless quantities (8).

In the imaginary time formulation of quantum field theory, the Green functions look like the correlation functions used in statistical mechanics. The partition function has the form

$$
Z=\int D \varphi_{C}(\mathbf{x}) e^{-S_{E}\left[\varphi_{C}\right]},
$$

where the integration measure $D \varphi_{C}$ is formally defined by

$$
D \varphi_{C}=\prod_{\mathbf{x}} d \varphi_{C}(\mathbf{x})
$$

Most of the variables of the system can be expressed in terms of the partition function or its derivatives.
2.3. Continuum Fractional Derivatives of the Riesz Type. To formulate a fractional generalization of the quantum field theory, we define fractional-order derivatives with respect to dimensionless Euclidean coordinates $x_{\mu}$, where $\mu=1,2,3,4$. These derivatives will be denoted by $\mathbb{D}_{C}^{ \pm}\left[\begin{array}{l}\alpha \\ \mu\end{array}\right]$, where $\alpha$ is the order of the derivative; $\mu$ denotes the coordinate $x_{\mu}$ with respect to which the derivative is taken, $C$ marks that the derivative is used for continuum field theory ( $L$ will be used for lattice operators), and + and - denote the even and odd types of the derivatives.

Definition 1. Continuum fractional derivatives $\mathbb{D}_{C}^{+}\left[\begin{array}{l}\alpha \\ \mu\end{array}\right]$ of the Riesz type and noninteger order $\alpha>0$ are defined by the equation

$$
\begin{array}{r}
\mathbb{D}_{C}^{+}\left[\begin{array}{l}
\alpha \\
\mu
\end{array}\right] \varphi_{C}(\mathbf{x})=\frac{1}{d_{1}(m, \alpha)} \int_{\mathbb{R}^{1}} \frac{1}{\left|z_{\mu}\right|^{\alpha+1}}\left(\Delta_{z_{\mu}}^{m} \varphi\right)(\mathbf{x}) d z_{\mu,} \\
(0<\alpha<m),
\end{array}
$$

where $m$ is the integer number that is greater than $\alpha$ and the operators $\left(\Delta_{z_{\mu}}^{m} \varphi\right)(\mathbf{x})$ are a finite difference $[1,2]$ of order $m$ of a function $\varphi_{C}(\mathbf{x})$ with the vector step $\mathbf{z}_{\mu}=z_{\mu} \mathbf{e}_{\mu} \in \mathbb{R}^{4}$ for the point $\mathbf{x} \in \mathbb{R}^{4}$. The centered difference

$$
\left(\Delta_{z_{\mu}}^{m} \varphi\right)\left(\mathbf{x}_{\mu}\right)=\sum_{n=0}^{m}(-1)^{n} \frac{m !}{n !(m-n) !} \varphi\left(\mathbf{x}-\left(\frac{m}{2}-n\right) z_{\mu} \mathbf{e}_{\mu}\right) .
$$

The constant $d_{1}(m, \alpha)$ is defined by

$$
d_{1}(m, \alpha)=\frac{\pi^{3 / 2} A_{m}(\alpha)}{2^{\alpha} \Gamma(1+\alpha / 2) \Gamma((1+\alpha) / 2) \sin (\pi \alpha / 2)},
$$

where

$$
A_{m}(\alpha)=2 \sum_{j=0}^{[m / 2]}(-1)^{j-1} \frac{m !}{j !(m-j) !}\left(\frac{m}{2}-j\right)^{\alpha}
$$

for the centered difference (15).

The constants $d_{1}(m, \alpha)$ are different from zero for all $\alpha>$ 0 in the case of an even $m$ and centered difference $\left(\Delta_{i}^{m} u\right)$ (see Theorem 26.1 in [1]). Note that the integral (14) does not depend on the choice of $m>\alpha$. Therefore, we can always choose an even number $m$ so that it is greater than parameter $\alpha$, and we can use the centered difference (15) for all positive real values of $\alpha$.

Using (14), we can see that the continuum fractional derivative $\mathbb{D}_{C}^{+}\left[\begin{array}{l}\alpha \\ \mu\end{array}\right]$ is the Riesz derivative that acts on the field $\varphi_{C}(\mathbf{x})$ with respect to the component $x_{\mu} \in \mathbb{R}^{1}$ of the vector $\mathbf{x} \in \mathbb{R}^{4}$; that is, the operator $\mathbb{D}_{C}^{+}\left[\begin{array}{l}\alpha \\ \mu\end{array}\right]$ can be considered as a partial fractional derivative of Riesz type.

The Riesz fractional derivatives for even $\alpha=2 m$, where $m \in \mathbb{N}$, are connected with the usual partial derivative of integer orders $2 m$ by the relation

$$
\mathbb{D}_{C}^{+}\left[\begin{array}{c}
2 m \\
\mu
\end{array}\right] \varphi_{C}(\mathbf{x})=(-1)^{m} \frac{\partial^{2 m} \varphi_{C}(\mathbf{x})}{\partial x_{\mu}^{2 m}} .
$$


The fractional derivatives $\mathbb{D}_{C}^{+}\left[\begin{array}{c}2 m \\ \mu\end{array}\right]$ for even orders $\alpha$ are local operators. Note that the Riesz derivative $\mathbb{D}_{C}^{+}\left[\begin{array}{l}1 \\ \mu\end{array}\right]$ cannot be considered as a derivative of first order with respect to $x_{\mu}$; that is,

$$
\mathbb{D}_{C}^{+}\left[\begin{array}{l}
1 \\
\mu
\end{array}\right] \varphi_{C}(\mathbf{x}) \neq \frac{\partial \varphi_{C}(\mathbf{x})}{\partial x_{\mu}}
$$

For $\alpha=1$ the operator $\mathbb{D}_{C}^{+}\left[\begin{array}{l}1 \\ \mu\end{array}\right]$ is nonlocal like a "square root of the Laplacian." Note that the Riesz derivatives for odd orders $\alpha=2 m+1$, where $m \in \mathbb{N}$, are nonlocal operators that cannot be considered as usual derivatives $\partial^{2 m+1} / \partial x^{2 m+1}$.

An important property of the Riesz fractional derivatives is the Fourier transform $\mathscr{F}$ of these operators in the form

$$
\mathscr{F}\left(\mathbb{D}_{C}^{+}\left[\begin{array}{l}
\alpha \\
\mu
\end{array}\right] \varphi_{C}(\mathbf{x})\right)(\mathbf{k})=\left|k_{\mu}\right|^{\alpha}(\mathscr{F} \varphi)(\mathbf{k}) \text {. }
$$

Property (20) is valid for functions $\varphi_{C}(\mathbf{x})$ from the space of infinitely differentiable functions with compact support. It also holds for the Lizorkin space (see Section 8.1 in [1]).

Let us consider the continuum fractional derivative $\mathbb{D}_{C}^{-}\left[\begin{array}{l}\alpha \\ \mu\end{array}\right]$ of the Riesz type that has the property

$$
\mathscr{F}\left(\mathbb{D}_{C}^{-}\left[\begin{array}{l}
\alpha \\
\mu
\end{array}\right] \varphi_{C}(\mathbf{x})\right)(\mathbf{k})=i \operatorname{sgn}\left(k_{\mu}\right)\left|k_{\mu}\right|^{\alpha}(\mathscr{F} \varphi)(\mathbf{k})
$$

$(\alpha>0)$

where $\operatorname{sgn}\left(k_{\mu}\right)$ is the sign function that extracts the sign of a real number $\left(k_{\mu}\right)$. For $0<\alpha<1$ the operator $\mathbb{D}_{C}^{-}\left[\begin{array}{l}\alpha \\ \mu\end{array}\right]$ can be considered as the conjugate Riesz derivative [30] with respect to $x_{\mu}$. Therefore, the operator (21) will be called a generalized conjugate derivative of the Riesz type.

The fractional operator $\mathbb{D}_{C}^{-}\left[\begin{array}{l}\alpha \\ \mu\end{array}\right]$ will be defined separately for the following three cases: (a) $\alpha>1$; (b) $\alpha=1$; (c) $0<\alpha<$ 1 .

Definition 2. Continuum fractional derivatives $\mathbb{D}_{C}^{+}\left[\begin{array}{l}\alpha \\ \mu\end{array}\right]$ of the Riesz type are defined by the following equations.

(a) For $\alpha>1$, the fractional operator $\mathbb{D}_{C}^{+}\left[\begin{array}{l}\alpha \\ \mu\end{array}\right]$ is defined by the equation

$$
\begin{aligned}
& \mathbb{D}_{C}^{-}\left[\begin{array}{l}
\alpha \\
j
\end{array}\right] \varphi_{C}(\mathbf{x}) \\
& =\frac{1}{d_{1}(m, \alpha-1)} \frac{\partial}{\partial x_{\mu}} \int_{\mathbb{R}^{1}} \frac{1}{\left|z_{\mu}\right|^{\alpha}}\left(\Delta_{z_{\mu}}^{m} \varphi\right)(\mathbf{x}) d z_{\mu}, \\
& \quad(1<\alpha<m+1),
\end{aligned}
$$

where $\left(\Delta_{z_{\mu}}^{m} \varphi\right)(\mathbf{x})$ is a finite difference that is defined in (15).

(b) For integer values $\alpha=1$, we have

$$
\mathbb{D}_{C}^{-}\left[\begin{array}{l}
1 \\
\mu
\end{array}\right] \varphi_{C}(\mathbf{x})=\frac{\partial \varphi_{C}(\mathbf{x})}{\partial x_{\mu}}
$$

(c) For $0<\alpha<1$, the fractional operator $\mathbb{D}_{C}^{+}\left[\begin{array}{l}\alpha \\ \mu\end{array}\right]$ is defined by the equation

$$
\begin{aligned}
& \mathbb{D}_{C}^{-}\left[\begin{array}{l}
\alpha \\
\mu
\end{array}\right] \varphi_{C}(\mathbf{x}) \\
& \quad=\frac{\partial}{\partial x_{\mu}} \int_{\mathbb{R}^{1}} R_{1-\alpha}\left(x_{\mu}-z_{\mu}\right) \varphi\left(\mathbf{x}+\left(z_{\mu}-x_{\mu}\right) \mathbf{e}_{\mu}\right) d z_{\mu},
\end{aligned}
$$$$
(0<\alpha<1) \text {, }
$$

where $\mathbf{e}_{\mu}$ is the basis of the Cartesian coordinate system, the function $R_{\alpha}(x)$ is the Riesz kernel that is defined by

$$
R_{\alpha}(x)= \begin{cases}\gamma_{1}^{-1}(\alpha)|x|^{\alpha-1} & \alpha \neq 2 n+1, n \in \mathbb{N} \\ -\gamma_{1}^{-1}(\alpha)|x|^{\alpha-1} \ln |x| & \alpha=2 n+1, n \in \mathbb{N}\end{cases}
$$

and the constant $\gamma_{1}(\alpha)$ has the form

$\gamma_{1}(\alpha)$

$$
= \begin{cases}\frac{2^{\alpha} \pi^{1 / 2} \Gamma(\alpha / 2)}{\Gamma((1-\alpha) / 2)} & \alpha \neq 2 n+1, \\ (-1)^{(1-\alpha) / 2} 2^{\alpha-1} \pi^{1 / 2} \Gamma\left(\frac{\alpha}{2}\right) \Gamma\left(1+\frac{[\alpha-1]}{2}\right) & \alpha=2 n+1,\end{cases}
$$

with $n \in \mathbb{N}$ and $\alpha \in \mathbb{R}_{+}$.

Note that the distinction between the continuum fractional derivatives $\mathbb{D}_{C}^{-}\left[\begin{array}{l}\alpha \\ \mu\end{array}\right]$ and the Riesz 4-dimensional fractional derivative consists [2] in the use of $\left|k_{\mu}\right|^{\alpha}$ instead of $|\mathbf{k}|^{\alpha}$.

For integer odd values of $\alpha$, we have

$$
\begin{array}{r}
\mathbb{D}_{C}^{-}\left[\begin{array}{c}
2 m+1 \\
\mu
\end{array}\right] \varphi_{C}(\mathbf{x})=(-1)^{m} \frac{\partial^{2 m+1} \varphi_{C}(\mathbf{x})}{\partial x_{\mu}^{2 m+1}}, \\
(m \in \mathbb{N}) .
\end{array}
$$

Equation (27) means that the fractional derivatives $\mathbb{D}_{C}^{-}\left[\begin{array}{l}\alpha \\ \mu\end{array}\right]$ of the odd orders $\alpha$ are local operators represented by the usual derivatives of integer orders. Note that the continuum derivative $\mathbb{D}_{C}^{-}\left[\begin{array}{c}2 m \\ \mu\end{array}\right]$ with $m \in \mathbb{N}$ cannot be considered as a local derivative of the order $2 m$ with respect to $x_{\mu}$. For $\alpha=2$ the generalized conjugate Riesz derivative is not the local derivative $\partial^{2} / \partial^{2} x_{\mu}$. The derivatives $\mathbb{D}_{C}^{-}\left[\begin{array}{l}\alpha \\ \mu\end{array}\right]$ for even orders $\alpha=2 m$, where $m \in \mathbb{N}$, are nonlocal operators that cannot be considered as usual derivatives $\partial^{2 m} / \partial x_{\mu}^{2 m}$.

It is important to note that the usual Leibniz rule for the derivative of products of two or more functions does not hold for derivatives of noninteger orders and for integer orders different from one [31]. This violation of the usual Leibniz rule is a characteristic property of all types of fractional derivatives.

Equations (18) and (27) allow us to state that the partial derivatives of integer orders are obtained from the fractional 
derivatives of the Riesz type $\mathbb{D}_{C}^{ \pm}\left[\begin{array}{l}\alpha \\ \mu\end{array}\right]$ for odd values $\alpha=2 m j+$ $1>0$ by $\mathbb{D}_{C}^{-}\left[\begin{array}{l}\alpha \\ \mu\end{array}\right]$ only, and for even values $\alpha=2 m>0(m \in$ $\mathbb{N})$, by $\mathbb{D}_{C}^{+}\left[\begin{array}{l}\alpha \\ \mu\end{array}\right]$. The continuum derivatives of the Riesz type $\mathbb{D}_{C}^{-}\left[\begin{array}{c}2 m \\ \mu\end{array}\right]$ and $\mathbb{D}_{C}^{+}\left[\begin{array}{c}2 m+1 \\ \mu\end{array}\right]$ are nonlocal differential operators of integer orders.

In formulation of fractional analogs of classical field theories, we need to generalize some field equations with partial differential equations of integer order. It is obvious that we would like to have a fractional generalization of these integerorder differential equations so as to obtain the original equations in the limit case, when the orders of generalized derivatives become equal to initial integer values. In order for this requirement to hold we can use the following rules of generalization:

$$
\begin{array}{r}
\frac{\partial^{2 m}}{\partial x_{\mu}^{2 m}}=(-1)^{m} \mathbb{D}_{C}^{+}\left[\begin{array}{c}
2 m \\
\mu
\end{array}\right] \rightarrow(-1)^{m} \mathbb{D}_{C}^{+}\left[\begin{array}{l}
\alpha \\
\mu
\end{array}\right], \\
(m \in \mathbb{N}, 2 m-1<\alpha<2 m+1), \\
\frac{\partial^{2 m+1}}{\partial x_{\mu}^{2 m+1}}=(-1)^{m} \mathbb{D}_{C}^{-}\left[\begin{array}{c}
2 j+1 \\
\mu
\end{array}\right] \rightarrow(-1)^{m} \mathbb{D}_{C}^{-}\left[\begin{array}{l}
\alpha \\
\mu
\end{array}\right], \\
(m \in \mathbb{N}, 2 m<\alpha<2 m+2) .
\end{array}
$$

In order to derive a fractional generalization of differential equation with partial derivatives of integer orders, we should replace the usual derivatives of odd orders with respect to $x_{\mu}$ by the continuum fractional derivatives $\mathbb{D}_{C}^{-}\left[\begin{array}{l}\alpha \\ \mu\end{array}\right]$ and the usual derivatives of even orders with respect to $x_{\mu}$ by the continuum fractional derivatives of the Riesz type $\mathbb{D}_{C}^{+}\left[\begin{array}{l}\alpha \\ \mu\end{array}\right]$.

2.4. Continuum Fractional 4-Dimensional Laplacian. The 4dimensional Laplacian $\square_{E, C}$ is defined by (10) as an operator of second order for Euclidean space-time.

Fractional-order generalizations of the d'Alembert operator $\square$ and the $N$-dimensional Laplacian $\square_{E}$ are considered in [14] and in Section 28 of [1].

It is important to note that an action of two repeated fractional derivatives of order $\alpha$ is not equivalent to the action of the fractional derivative of the double order $2 \alpha$,

$$
\mathbb{D}_{C}^{ \pm}\left[\begin{array}{l}
\alpha \\
\mu
\end{array}\right] \mathbb{D}_{C}^{ \pm}\left[\begin{array}{l}
\alpha \\
\mu
\end{array}\right] \neq \mathbb{D}_{C}^{ \pm}\left[\begin{array}{c}
2 \alpha \\
\mu
\end{array}\right], \quad(\alpha>0) .
$$

The continuum 4-dimensional Laplacian of noninteger order for the scalar field $\varphi_{C}(\mathbf{x})$ can be defined by two different equations, where the first expression contains the two lattice operators of order $\alpha$, and the second expression contains the fractional derivatives of the doubled order $2 \alpha$.

Definition 3. The continuum 4-dimensional Laplace operators $\square_{E, C}^{\alpha, \alpha, \pm}$ and $\square_{E, C}^{2 \alpha, \pm}$ of noninteger order $2 \alpha$ for the scalar field $\varphi_{C}(\mathbf{x})$ are defined by the different equations:

$$
\square_{E, C}^{\alpha, \alpha, \pm} \varphi_{C}(\mathbf{x})=\sum_{\mu=1}^{4}\left(\mathbb{D}_{C}^{ \pm}\left[\begin{array}{l}
\alpha \\
\mu
\end{array}\right]\right)^{2} \varphi_{C}(\mathbf{x})
$$

$$
\square_{E, C}^{2 \alpha, \pm} \varphi_{L}(\mathbf{x})=\sum_{\mu=1}^{4} \mathbb{D}_{C}^{ \pm}\left[\begin{array}{c}
2 \alpha \\
\mu
\end{array}\right] \varphi_{C}(\mathbf{x}),
$$

where $\mathbb{D}_{C}^{ \pm}$are defined in Definitions 1 and 2.

The violation of the semigroup property (29) leads to the fact that the operators (30) and (31) do not coincide in general.

It should be noted that the operators $\square_{E, C}^{\alpha, \alpha,-}$ and $\square_{E, C}^{2 \alpha,+}$ for integer $\alpha=1$ gives the usual (local) 4-dimensional Laplacian $\square_{E}$ that is defined by (7); that is,

$$
\square_{E, C}^{1,1,-}=\square_{E, C}^{2,+}=\square_{E} .
$$

The operators $\square_{E, C}^{\alpha, \alpha,+}$ and $\square_{E, C}^{2 \alpha,-}$ for integer $\alpha=1$ are nonlocal operators of the second orders that cannot be considered as $\square_{E}$ :

$$
\square_{E, C}^{1,1,+} \neq \square_{E}, \quad \square_{E, C}^{2,-} \neq \square_{E} .
$$

Therefore we should use only the continuum fractional 4dimensional Laplace operators $\square_{E, C}^{\alpha, \alpha,-}$ or $\square_{E, C}^{2 \alpha,+}$ in the fractional field theory, since the operators $\square_{E, C}^{\alpha, \alpha,+}$ or $\square_{E, C}^{2 \alpha,-}$ do not satisfy the correspondence principle for $\alpha=1$.

Fractional Laplace operators have been suggested by Riesz in [14] for the first time. The fractional Laplacian $(-\Delta)_{C}^{\alpha / 2}$ in the Riesz form for 4-dimensional Euclidean spacetime $\mathbb{R}^{4}$ can be considered as an inverse Fourier's integral transform $\mathscr{F}^{-1}$ of $|\mathbf{k}|^{\alpha}$ by

$$
\left((-\Delta)_{C}^{\alpha / 2} \varphi\right)(\mathbf{x})=\mathscr{F}^{-1}\left(|\mathbf{k}|^{\alpha}(\mathscr{F} \varphi)(\mathbf{k})\right),
$$

where $\alpha>0$ and $\mathbf{x} \in \mathbb{R}^{4}$.

Definition 4. For $\alpha>0$, the fractional Laplacian of the Riesz form is defined as the hypersingular integral

$$
\left((-\Delta)_{C}^{\alpha / 2} \varphi_{C}\right)(\mathbf{x})=\frac{1}{d_{4}(m, \alpha)} \int_{\mathbb{R}^{4}} \frac{1}{|\mathbf{z}|^{\alpha+4}}\left(\Delta_{\mathbf{z}}^{m} \varphi_{C}\right)(\mathbf{z}) d^{4} \mathbf{z},
$$

where $m>\alpha$ and $\left(\Delta_{\mathbf{z}}^{m} \varphi\right)(\mathbf{z})$ is a finite difference of order $m$ of a field $\varphi_{C}(\mathbf{x})$ with a vector step $\mathbf{z} \in \mathbb{R}^{4}$ and centered at the point $\mathbf{x} \in \mathbb{R}^{4}$ :

$$
\left(\Delta_{\mathbf{z}}^{m} \varphi\right)(\mathbf{z})=\sum_{j=0}^{m}(-1)^{j} \frac{m !}{j !(m-j) !} \varphi(\mathbf{x}-j \mathbf{z}) .
$$

The constant $d_{4}(m, \alpha)$ is defined by

$$
d_{4}(m, \alpha)=\frac{\pi^{3} A_{m}(\alpha)}{2^{\alpha} \Gamma(1+\alpha / 2) \Gamma(2+\alpha / 2) \sin (\pi \alpha / 2)},
$$

where

$$
A_{m}(\alpha)=\sum_{j=0}^{m}(-1)^{j-1} \frac{m !}{j !(m-j) !} j^{\alpha} .
$$

Note that the hypersingular integral (35) does not depend on the choice of $m>\alpha$. The Fourier transform $\mathscr{F}$ of the fractional Laplacian is given by $\mathscr{F}\left\{(-\Delta)_{C}^{\alpha / 2} \varphi\right\}(\mathbf{k})=$ $|\mathbf{k}|^{\alpha}(\mathscr{F} \varphi)(\mathbf{k})$. This equation is valid for the Lizorkin space [1] 
and the space $C^{\infty}\left(\mathbb{R}^{4}\right)$ of infinitely differentiable functions on $\mathbb{R}^{4}$ with compact support.

2.5. Fractional Field Equations. The Euclidean action $S_{E}\left[\varphi_{C}\right]$ for fractional scalar fields can be defined by the expression

$$
\begin{aligned}
& S_{E}^{(\alpha)}\left[\varphi_{C}, J_{C}\right] \\
& =\frac{1}{2} \int d^{4} \mathbf{x} \varphi_{C}(\mathbf{x})\left(\square_{E, C}^{2 \alpha,+}+M_{C}^{2}\right) \varphi_{C}(\mathbf{x})+\int d^{4} \mathbf{x} J_{C}(\mathbf{x}) \varphi_{C}(\mathbf{x}),
\end{aligned}
$$

where $\square_{E, C}^{2 \alpha,+}$ denotes the fractional 4-dimensional Laplacian (31) for dimensionless variables $\mathbf{x}$ of continuum space-time. Here we take into account (18) in the form $\square_{E, C}^{2,+}=-\square_{E, C}$.

Using the stationary action principle, $\delta S_{E}^{(\alpha)}\left[\varphi_{C}, J_{C}\right]=0$, we derive the fractional field equation

$$
\left(\square_{E, C}^{2 \alpha,+}+M_{C}^{2}\right) \varphi_{C}(\mathbf{x})=J_{C}(\mathbf{x}) .
$$

Similarly, we can consider the fractional field theories that are described by the fractional field equations

$$
\begin{gathered}
\left(\square_{E, C}^{\alpha, \alpha,-}+M_{C}^{2}\right) \varphi_{C}(\mathbf{x})=J_{C}(\mathbf{x}), \\
\left((-\Delta)_{C}^{\alpha / 2}+M_{C}^{2}\right) \varphi_{C}(\mathbf{x})=J_{C}(\mathbf{x}),
\end{gathered}
$$

where the fractional 4-dimensional Laplacians (30) and (35) are used.

The Green functions $G_{s, C, E}^{(\alpha)}\left(\mathbf{x}_{1}, \ldots, \mathbf{x}_{s}\right)=\left\langle\varphi_{C}\left(\mathbf{x}_{1}\right) \ldots\right.$ $\left.\varphi_{C}\left(\mathbf{x}_{s}\right)\right\rangle_{E}^{(\alpha)}$ for Euclidean space-time and dimensionless variables have the following path integral representation:

$$
G_{s, C, E}^{(\alpha)}\left(\mathbf{x}_{1}, \ldots, \mathbf{x}_{s}\right)=\frac{\int D \varphi_{C}\left(\varphi_{C}\left(\mathbf{x}_{1}\right) \cdots \varphi_{C}\left(\mathbf{x}_{s}\right)\right) e^{-S_{E}^{(\alpha)}\left[\varphi_{C}, J_{C}\right]}}{\int D \varphi_{C} e^{-S_{E}^{(\alpha)}\left[\varphi_{C}, J_{C}\right]}},
$$

where $\int D \varphi_{C}$ is the sum over all possible configurations of the field $\varphi_{C}(\mathbf{x})$ for continuum space-time. Note that the pathintegral approach for space-fractional quantum mechanics is considered in $[3,4,32]$.

The Euclidean Green functions (42) of fractional field theory can be derived from the generating functional

$$
Z_{0, C}^{(\alpha)}\left[J_{C}\right]=\int D \varphi_{C} e^{-S_{E}^{(\alpha)}\left[\varphi_{C}, J_{C}\right]} .
$$

Using the integer-order differentiation of (43) with respect to the sources $J_{n}$, we can obtain the correlation functions. The $s$-point fractional correlation function is

$$
\left\langle\varphi_{C}\left(\mathbf{x}_{1}\right) \cdots \varphi_{C}\left(\mathbf{x}_{s}\right)\right\rangle_{E}^{(\alpha)}=\frac{\delta^{s} Z_{0, C}^{(\alpha)}\left[J_{C}\right]}{\delta J_{C}\left(\mathbf{x}_{1}\right) \cdots \delta J_{C}\left(\mathbf{x}_{s}\right)} .
$$

Quantum fluctuations correspond to the contributions to the integral (43) coming from field configurations which are not solutions to the classical field equations (40) and (41).

\section{Fractional Field Theory on Lattice Space-Time}

3.1. Lattice Space-Time. In quantum field theory, a lattice approach is based on lattice space-time instead of the continuum of space-time. Lattice models originally occurred in the condensed matter physics, where the atoms of a crystal form a lattice. The unit cell is represented in terms of the lattice parameters, which are the lengths of the cell edges $\left(\mathbf{a}_{\mu}\right.$, where $\mu=1,2,3,4)$ and the angles between them.

Let us consider an unbounded space-time lattice characterized by the noncoplanar vectors $\mathbf{a}_{\mu}, \mu=1,2,3,4$, that are the shortest vectors by which a lattice can be displaced and be brought back into itself. For simplification, we assume that $\mathbf{a}_{\mu}, \mu=1,2,3,4$, are mutually perpendicular primitive lattice vectors. We choose directions of the axes of the Cartesian coordinate system coinciding with the vector $\mathbf{a}_{\mu}$. Then $\mathbf{a}_{\mu}=$ $a_{\mu} \mathbf{e}_{\mu}$, where $a_{\mu}=\left|\mathbf{a}_{\mu}\right|$, and $\mathbf{e}_{\mu},(\mu=1,2,3,4)$, are the basis vectors of the Cartesian coordinate system for Euclidean space-time $\mathbb{R}^{4}$. This simplification means that the lattice is a primitive 4-dimensional orthorhombic Bravais lattice. The position vector of an arbitrary lattice site is written as

$$
\mathbf{x}(\mathbf{n})=\sum_{\mu=1}^{4} n_{\mu} \mathbf{a}_{\mu}
$$

where $n_{\mu}$ are integer. In a lattice the sites are numbered by $\mathbf{n}$, so that the vector $\mathbf{n}=\left(n_{1}, n_{2}, n_{4}, n_{4}\right)$ can be considered as a number vector of the corresponding lattice site.

As the lattice fields we consider real-valued functions for $\mathbf{n}$-sites. For simplification, we consider the scalar field $\varphi_{L}(\mathbf{n})$ for lattice sites that is defined by $\mathbf{n}=\left(n_{1}, n_{2}, n_{3}, n_{4}\right)$. In many cases, we can assume that $\varphi_{L}(\mathbf{n})$ belongs to the Hilbert space $l_{2}$ of square-summable sequences to apply the discrete Fourier transform. For simplification, we will consider operators for the lattice scalar fields $\varphi_{L}(\mathbf{n})=\varphi\left(n_{1}, n_{2}, n_{3}, n_{4}\right)$. All consideration can be easily generalized to the case of the vector fields and other types of fields.

For continuum fractional field theory, we use the dimensionless quantities (8). In the lattice fractional theory, we also will be using the physically dimensionless quantities such as $a_{\mu}, n_{\mu}, \mathbf{x}(\mathbf{n}), \mathbf{e}_{\mu}$, and $\varphi_{L}(\mathbf{n})$.

3.2. Lattice Fractional Derivative. Let us give a definition of lattice partial derivative $\mathbb{D}_{L}^{ \pm}\left[\begin{array}{c}\alpha \\ \mu\end{array}\right]$ of arbitrary positive real order $\alpha$ in the direction $\mathbf{e}_{\mu}=\mathbf{a}_{\mu} /\left|\mathbf{a}_{\mu}\right|$ in the lattice spacetime.

Definition 5. Lattice fractional partial derivatives are the operators $\mathbb{D}_{L}^{ \pm}\left[\begin{array}{c}\alpha \\ \mu\end{array}\right]$ such that

$$
\begin{array}{r}
\left(\mathbb{D}_{L}^{ \pm}\left[\begin{array}{l}
\alpha \\
\mu
\end{array}\right] \varphi_{L}\right)(\mathbf{n})=\frac{1}{a_{\mu}^{\alpha}} \sum_{m_{\mu}=-\infty}^{+\infty} K_{\alpha}^{ \pm}\left(n_{\mu}-m_{\mu}\right) \varphi_{L}(\mathbf{m}), \\
(\mu=1,2,3,4),
\end{array}
$$


where $\alpha \in \mathbb{R}, \alpha>0, n_{\mu}, m_{\mu} \in \mathbb{Z}$, and the kernels $K_{\alpha}^{ \pm}(n-m)$ are defined by the equations

$$
K_{\alpha}^{+}(n-m)=\frac{\pi^{\alpha}}{\alpha+1}{ }_{1} F_{2}\left(\frac{\alpha+1}{2} ; \frac{1}{2}, \frac{\alpha+3}{2} ;-\frac{\pi^{2}(n-m)^{2}}{4}\right),
$$

$$
\begin{aligned}
& K_{\alpha}^{-}(n-m) \\
& =-\frac{\pi^{\alpha+1}(n-m)}{\alpha+2}{ }_{1} F_{2}\left(\frac{\alpha+2}{2} ; \frac{3}{2}, \frac{\alpha+4}{2} ;-\frac{\pi^{2}(n-m)^{2}}{4}\right),
\end{aligned}
$$$$
\alpha>0,
$$

where ${ }_{1} F_{2}$ is the Gauss hypergeometric function [33, 34]. The parameter $\alpha>0$ will be called the order of the lattice derivatives (46).

The kernels $K_{\alpha}^{ \pm}(n)$ are real-valued functions of integer variable $n \in \mathbb{Z}$. The kernel $K_{\alpha}^{+}(n)$ is even function $K_{\alpha}^{+}(-n)=$ $+K_{\alpha}^{+}(n)$, and $K_{\alpha}^{-}(n)$ is odd function $K_{\alpha}^{-}(-n)=-K_{\alpha}^{-}(n)$ for all $n \in \mathbb{Z}$.

The reasons to define the kernels $K_{\alpha}^{ \pm}(n-m)$ in the forms (47) and (48) are based on the expressions of their Fourier series transforms. The Fourier series transform

$$
\widehat{K}_{\alpha}^{+}(k)=\sum_{n=-\infty}^{+\infty} e^{-i k n} K_{\alpha}^{+}(n)=2 \sum_{n=1}^{\infty} K_{\alpha}^{+}(n) \cos (k n)+K_{\alpha}^{+}(0)
$$

for the kernel $K_{\alpha}^{+}(n)$ defined by (47) satisfies the condition

$$
\widehat{K}_{\alpha}^{+}(k)=|k|^{\alpha}, \quad(\alpha>0) .
$$

The Fourier series transforms

$$
\widehat{K}_{\alpha}^{-}(k)=\sum_{n=-\infty}^{+\infty} e^{-i k n} K_{\alpha}^{-}(n)=-2 i \sum_{n=1}^{\infty} K_{\alpha}^{-}(n) \sin (k n)
$$

for the kernels $K_{\alpha}^{-}(n)$ defined by (48) satisfies the condition

$$
\widehat{K}_{\alpha}^{-}(k)=i \operatorname{sgn}(k)|k|^{\alpha}, \quad(\alpha>0) .
$$

Note that we use the minus sign in the exponents of (49) and (51) instead of plus in order to have the plus sign for plane waves and for the Fourier series.

The form (47) of the kernel $K_{\alpha}^{+}(n-m)$ is completely determined by the requirement (50). If we use an inverse relation of (49) with $\widehat{K}_{\alpha}^{+}(k)=|k|^{\alpha}$ that has the form

$$
K_{\alpha}^{+}(n)=\frac{1}{\pi} \int_{0}^{\pi} k^{\alpha} \cos (n k) d k, \quad(\alpha \in \mathbb{R}, \alpha>0),
$$

then we get (47) for the kernel $K_{\alpha}^{+}(n-m)$. The form (48) of the term $K_{\alpha}^{-}(n-m)$ is completely determined by (52). Using the inverse relation of (51) with $\widehat{K}_{\alpha}^{-}(k)=i \operatorname{sgn}(k)|k|^{\alpha}$ in the form

$$
K_{\alpha}^{-}(n)=-\frac{1}{\pi} \int_{0}^{\pi} k^{\alpha} \sin (n k) d k \quad(\alpha \in \mathbb{R}, \alpha>0),
$$

we get (48) for the kernel $K_{\alpha}^{-}(n-m)$. Note that $K_{\alpha}^{-}(0)=0$.

The lattice operators (46) with (47) and (48) for integer and noninteger orders $\alpha$ can be interpreted as a long-range interactions of the lattice site defined by $n$ with all other sites with $m \neq n$.

3.3. Lattice Operators of Integer Orders. Let us give exact forms of the kernels $\widehat{K}_{\alpha}^{ \pm}(k)$ for integer positive $\alpha \in \mathbb{N}$. Equations (47) and (48) for the case $\alpha \in \mathbb{N}$ can be simplified. To obtain the simplified expressions for kernels $\widehat{K}_{\alpha}^{ \pm}(k)$ with positive integer $\alpha=m$, we use the integrals of Sec. 2.5.3.5 in [35]. The kernels $K_{\alpha}^{ \pm}(n)$ for integer positive $\alpha=m$ are defined by the equations

$$
\begin{aligned}
K_{\alpha}^{+}(n)= & \sum_{k=0}^{[(\alpha-1) / 2]} \frac{(-1)^{n+k} s ! \pi^{\alpha-2 k-2}}{(\alpha-2 n-1) !} \frac{1}{n^{2 k+2}} \\
& +\frac{(-1)^{[(\alpha+1) / 2]} s !(2[(\alpha+1) / 2]-\alpha)}{\pi n^{\alpha+1}}, \\
K_{\alpha}^{-}(n)=- & \sum_{k=0}^{[\alpha / 2]} \frac{(-1)^{n+k+1} s ! \pi^{\alpha-2 k-1}}{(\alpha-2 n) !} \frac{1}{n^{2 k+2}} \\
& -\frac{(-1)^{[\alpha / 2]} s !(2[\alpha / 2]-\alpha+1)}{\pi n^{\alpha+1}},
\end{aligned}
$$

where $[x]$ is the integer part of the value $x$, and $n \in \mathbb{N}$. Here $2[(m+1) / 2]-m=1$ for odd $m$, and $2[(m+1) / 2]-m=0$ for even $m$.

Using (55) or direct integration (53) for integer values $\alpha=$ 1 and $\alpha=2$, we get the simplest examples of $K_{\alpha}^{+}(n)$ in the form

$$
K_{1}^{+}(n)=-\frac{1-(-1)^{n}}{\pi n^{2}}, \quad K_{2}^{+}(n)=\frac{2(-1)^{n}}{n^{2}},
$$

where $n \neq 0, n \in \mathbb{Z}$, and $K_{m}^{+}(0)=\pi^{m} /(m+1)$ for all $m \in \mathbb{N}$. Using (56) or direct integration (54) for $\alpha=1$ and $\alpha=2$, we get examples of $K_{\alpha}^{-}(n)$ in the form

$$
K_{1}^{-}(n)=\frac{(-1)^{n}}{n}, \quad K_{2}^{-}(n)=\frac{(-1)^{n} \pi}{n}+\frac{2\left(1-(-1)^{n}\right)}{\pi n^{3}},
$$

where $n \neq 0, n \in \mathbb{Z}$, and $K_{m}^{-}(0)=0$ for all $m \in \mathbb{N}$. Note that $\left(1-(-1)^{n}\right)=2$ for odd $n$, and $\left(1-(-1)^{n}\right)=0$ for even $n$.

In the definition of lattice fractional derivatives (46) the value $\mu=1,2,3,4$ characterizes the component $n_{\mu}$ of the lattice vector $\mathbf{n}$ with respect to which this derivative is taken. It is similar to the variable $x_{\mu}$ in the usual partial derivatives for the space-time $\mathbb{R}^{4}$. The lattice operators $\mathbb{D}_{L}^{ \pm}\left[\begin{array}{l}\alpha \\ \mu\end{array}\right]$ are analogous to the partial derivatives of order $\alpha$ with respect to coordinates $x_{\mu}$ for continuum field theory. The lattice derivative $\mathbb{D}_{L}^{ \pm}\left[\begin{array}{l}\alpha \\ \mu\end{array}\right]$ is an operator along the vector $\mathbf{e}_{\mu}=\mathbf{a}_{\mu} /\left|\mathbf{a}_{\mu}\right|$ in the lattice space-time. 
3.4. Lattice Operators with Other Kernels. In general, we can weaken the conditions (50) and (52) to determine a more wider class of the lattice fractional derivatives. For this aim, we replace the exact conditions (50) and (52) by the asymptotical requirements

$$
\begin{gathered}
\widehat{K}_{\alpha}^{+}(k)=|k|^{\alpha}+o\left(|k|^{\alpha}\right), \quad(k \longrightarrow 0), \\
\widehat{K}_{\alpha}^{-}(k)=i \operatorname{sgn}(k)|k|^{\alpha}+o\left(|k|^{\alpha}\right), \quad(k \longrightarrow 0),
\end{gathered}
$$

where the little-o notation $o\left(|k|^{\alpha}\right)$ means the terms that include higher powers of $|k|$ than $|k|^{\alpha}$. The conditions (59) and (60) mean that we can consider arbitrary functions $K_{\alpha}^{ \pm}(n-m)$ for which $\widehat{K}_{\alpha}^{ \pm}(k)$ are asymptotically equivalent to $|k|^{\alpha}$ and $i \operatorname{sgn}(k)|k|^{\alpha}$ as $|k| \rightarrow 0$, respectively.

As an example of the kernel $K_{\alpha}^{+}(n-m)$, which can give the lattice fractional derivatives (46) with (59), has been suggested in [18-20] in the form

$$
K_{\alpha}^{+}(n)=\frac{(-1)^{n} \Gamma(\alpha+1)}{\Gamma(\alpha / 2+1+n) \Gamma(\alpha / 2+1-n)},
$$

where we use relation 5.4.8.12 from [35]. This kernel has been suggested in $[18,19]$ to describe long-range interactions of the lattice particles for noninteger values of $\alpha$. For integer values of $\alpha \in \mathbb{N}$, the kernel $K_{\alpha}^{+}(n-m)=0$ for $|n-m| \geq \alpha / 2+$ 1. For $\alpha=2 j$, we have $K_{\alpha}^{+}(n-m)=0$ for all $|n-m| \geq$ $j+1$. The function $K_{\alpha}^{+}(n-m)$ with even value of $\alpha=2 j$ can be interpreted as an interaction of the $n$-particle with $2 j$ particles with numbers $n \pm 1 \cdots n \pm j$. Note that the long-range interaction with the kernel (61) is partially connected with the long-range interaction of the Grünwald-Letnikov-Riesz type [24]. It is easy to see that expression (47) is more complicated than (61).

As an example of the kernel $K_{\alpha}^{-}(n-m)$, which can give the lattice fractional derivatives (46) with (60), has been suggested in [20] in the form

$$
K_{\alpha}^{-}(n)=\frac{(-1)^{(n+1) / 2}(2[(n+1) / 2]-n) \Gamma(\alpha+1)}{2^{\alpha} \Gamma((\alpha+n) / 2+1) \Gamma((\alpha-n) / 2+1)},
$$

where the brackets [] mean the integral part, that is, the floor function that maps a real number to the largest previous integer number. The expression $(2[(n+1) / 2]-n)$ is equal to zero for even $n=2 m$, and it is equal to 1 for odd $n=2 m-1$. To get the expression, we use relation 5.4.8.13 from [35]. Note that the kernel (62) is real valued function since we have zero, when the expression $(-1)^{(n+1) / 2}$ becomes a complex number.

For $0<\alpha \leq 2$, we can give other examples of the kernels with the property (59) which are given in Section 8 of the book [36]. For example, the most frequently used kernel is

$$
K_{\alpha}^{+}(n)=\frac{A(\alpha)}{|n|^{\alpha+1}}
$$

where we use the multiplier $A(\alpha)=(2 \Gamma(-\alpha) \cos (\pi \alpha / 2))^{-1}$, which has the asymptotic behavior $\widehat{K}_{\alpha}^{+}(k)=\widehat{K}_{\alpha}^{+}(0)+|k|^{\alpha}+$ $o\left(|k|^{\alpha}\right),(k \rightarrow 0)$, for the cases $0<\alpha<2$ and $\alpha \neq 1$, with nonzero term $\widehat{K}_{\alpha}^{+}(0)$, where $\zeta(z)$ is the Riemann zetafunction. To take into account this expression, we use the asymptotic condition for $\widehat{K}_{\alpha}^{+}(k)$ in the form (50) that includes $\widehat{K}_{\alpha}^{+}(0)$. For details see Section 8.11-8.12 in [36].
3.5. Lattice Fractional 4-Dimensional Laplacian. An action of two repeated lattice operators of order $\alpha$ is not equivalent to the action of the lattice operator of double order $2 \alpha$ :

$$
\mathbb{D}_{L}^{ \pm}\left[\begin{array}{l}
\alpha \\
\mu
\end{array}\right] \mathbb{D}_{L}^{ \pm}\left[\begin{array}{l}
\alpha \\
\mu
\end{array}\right] \neq \mathbb{D}_{L}^{ \pm}\left[\begin{array}{c}
2 \alpha \\
\mu
\end{array}\right], \quad(\alpha>0) .
$$

Note that these properties are similar to noninteger order derivatives [2].

Definition 6. The lattice 4-dimensional fractional Laplacian operators $\square_{E, L}^{\alpha, \alpha, \pm}$ and $\square_{E, L}^{2 \alpha, \pm}$ for a scalar lattice field $\varphi_{L}(\mathbf{m})$ are defined by the following two equations, where the first expression contains the two lattice operators of order $\alpha$,

$$
\square_{E, L}^{\alpha, \alpha, \pm} \varphi_{L}(\mathbf{m})=\sum_{\mu=1}^{4}\left(\mathbb{D}_{L}^{ \pm}\left[\begin{array}{l}
\alpha \\
\mu
\end{array}\right]\right)^{2} \varphi_{L}(\mathbf{m}),
$$

and the second expression contains the lattice operator of the order $2 \alpha$ in the form

$$
\square_{E, L}^{2 \alpha, \pm} \varphi_{L}(\mathbf{m})=\sum_{\mu=1}^{4} \mathbb{D}_{L}^{ \pm}\left[\begin{array}{c}
2 \alpha \\
\mu
\end{array}\right] \varphi_{L}(\mathbf{m}) .
$$

The violation of the semigroup property (64) leads to the fact that operators (65) and (66) do not coincide in general.

Using (46), expression (66) can be represented by

$$
\left(\square_{E, L}^{2 \alpha, \pm} \varphi_{L}\right)(\mathbf{n})=\sum_{\mu=1}^{4} \frac{1}{a_{\mu}^{2 \alpha}} \sum_{m_{\mu}=-\infty}^{+\infty} K_{2 \alpha}^{ \pm}\left(n_{\mu}-m_{\mu}\right) \varphi_{L}(\mathbf{m}) .
$$

The correspondent continuum fractional Laplace operators are defined by (30) and (31). The continuum operators $\square_{E, C}^{\alpha, \alpha,-}$ and $\square_{E, C}^{2 \alpha,+}$ for integer $\alpha=1$ give the usual (local) 4dimensional Laplacian $\square_{E}$ that is defined by (7). The operators $\square_{E, C}^{\alpha, \alpha,+}$ and $\square_{E, C}^{2 \alpha,-}$ for integer $\alpha=1$ are nonlocal operators and cannot get a correspondence with the usual (nonfractional) field theories. Therefore we should use the lattice fractional Laplace operators $\square_{E, L}^{\alpha, \alpha,-}$ or $\square_{E, L}^{2 \alpha,+}$ in the lattice fractional field theories.

3.6. Lattice Riesz 4-Dimensional Laplacian. Let us define a lattice analog of the fractional Laplace operator of the Riesz type $[2,14]$ which is an operator for scalar fields on the lattice space-time.

Definition 7. The lattice fractional Laplace operator of the Riesz type $(-\Delta)_{L}^{\alpha / 2}$ for 4 -dimensional Euclidean space-time is defined by the equation

$$
\left((-\Delta)_{L}^{\alpha / 2} \varphi_{L}\right)(\mathbf{n})=\frac{1}{a^{\alpha}} \sum_{m_{1} \cdots m_{4}=-\infty}^{+\infty} \mathscr{K}_{\alpha}^{+}(\mathbf{n}-\mathbf{m}) \varphi_{L}(\mathbf{m}),
$$

where the constant $a$ is $a=\left(\sum_{\mu=1}^{4} a_{\mu}^{2}\right)^{\alpha / 2}$ and the kernel $\mathscr{K}_{\alpha}^{+}(\mathbf{n}-\mathbf{m})$ is defined by the equation

$$
\mathscr{K}_{\alpha}^{+}(\mathbf{n})=\frac{1}{\pi^{4}} \int_{0}^{\pi} d k_{1} \cdots \int_{0}^{\pi} d k_{4}\left(\sum_{\mu}^{4} k_{\mu}^{2}\right)^{\alpha / 2} \prod_{\mu=1}^{4} \cos \left(n_{\mu} k_{\mu}\right),
$$


where $\mathbf{n}=\sum_{\mu=1}^{4} n_{\mu} \mathbf{e}_{\mu}$ and the parameter $\alpha>0$ is the order of the lattice operator (68).

Note that the kernel (69) is connected with (47) by the equation

$$
\begin{aligned}
\frac{1}{\pi^{4}} & \int_{0}^{\pi} d k_{1} \cdots \int_{0}^{\pi} d k_{4}\left(k_{\mu}^{2}\right)^{\alpha / 2} \cos \left(n_{\mu} k_{\mu}\right) \\
& =\frac{\pi^{\alpha}}{\alpha+1}{ }_{1} F_{2}\left(\frac{\alpha+1}{2} ; \frac{1}{2}, \frac{\alpha+3}{2} ;-\frac{\pi^{2}\left(n_{\mu}\right)^{2}}{4}\right),
\end{aligned}
$$

where $\mathbf{n}_{\mu}=n_{\mu} \mathbf{e}_{\mu}$ without the sum over $\mu$.

The Fourier series transform $\widehat{\mathscr{K}}_{\alpha}^{+}(\mathbf{k})$ of the kernels $\mathscr{K}_{\alpha}^{+}(\mathbf{n})$ in the form

$$
\widehat{\mathscr{K}}_{\alpha}^{+}(\mathbf{k})=\sum_{n_{1} \cdots n_{4}=-\infty}^{+\infty} e^{-i \sum_{\mu=1}^{4} k_{\mu} n_{\mu}} \mathscr{K}_{\alpha}^{+}(\mathbf{n})
$$

satisfies the condition

$$
\widehat{\mathscr{K}}_{\alpha}^{+}(\mathbf{k})=|\mathbf{k}|^{\alpha}=\left(\sum_{\mu}^{4} k_{\mu}^{2}\right)^{\alpha / 2}, \quad(\alpha>0) .
$$

The form (69) of the kernel $\mathscr{K}_{\alpha}^{+}(\mathbf{n})$ is completely determined by the requirement (72). The inverse relation to (71) with (72) has the form (69).

If the lattice field $\varphi_{L}(\mathbf{m})$ depends only on one variable $m_{\mu}$ with fixed $\mu \in\{1,2,3,4\}$, that is, $\mathbf{m}=\mathbf{m}_{\mu}=m_{\mu} \mathbf{e}_{\mu}$ without the sum over $\mu$, then we have

$$
(-\Delta)_{L}^{\alpha / 2} \varphi_{L}\left(\mathbf{m}_{\mu}\right)=\mathbb{D}_{L}^{+}\left[\begin{array}{l}
\alpha \\
\mu
\end{array}\right] \varphi_{L}(\mathbf{m}) .
$$

The lattice fractional Laplacian $(-\Delta)_{L}^{\alpha / 2}$ in the Riesz form for 4-dimensional lattice space-time can be considered as a lattice analog of the fractional Laplacian $(-\Delta)_{C}^{\alpha / 2}$ for continuum Euclidean space-time $\mathbb{R}^{4}$ that is defined by (35).

3.7. Lattice Fractional Field Theory. The path integral (11) does not have a precise mathematical definition. To give a definition of the path integrals, we can introduce a space-time lattice with "lattice constants" $\mathbf{a}_{\mu}$. Every point on the lattice is then specified by four integers which are denoted by the vector $\mathbf{n}=\left(n_{1}, n_{2}, n_{3}, n_{4}\right)$, where the last component will denote a lattice analog of the Euclidean time.

In the path integral expression for lattice fields, we should use dimensionless variables only. Note that by convention all variables of the lattice theory are dimensionless variables.

For lattice fractional fied theory the path-integral expression of the Green functions is

$$
\begin{aligned}
& \left\langle\varphi_{L}\left(\mathbf{n}_{1}\right) \cdots \varphi_{L}\left(\mathbf{n}_{s}\right)\right\rangle \\
& =\frac{\int \prod_{j=1}^{s} d \varphi_{L}\left(\mathbf{n}_{j}\right)\left(\varphi_{L}\left(\mathbf{n}_{1}\right) \cdots \varphi_{L}\left(\mathbf{n}_{s}\right)\right) e^{-S_{E}\left[\varphi_{L}, J_{L}\right]}}{\int \prod_{i=1}^{s} d \varphi_{L}\left(\mathbf{n}_{i}\right) e^{-S_{E}\left[\varphi_{L}, J_{L}\right]}} .
\end{aligned}
$$

The structure of the path integral (74) is analogous to that used in the statistical mechanics of lattice system.
The lattice action $S_{E}\left[\varphi_{L}, J_{L}\right]$ is not unique, and we can choose the simplest one. We have only the requirement that any lattice action should reproduce the correct continuum expression in the continuum limit $a_{\mu} \rightarrow+0$.

The action used in the path integral (74) can be considered in the forms

$$
\begin{aligned}
S_{E}\left[\varphi_{L}, J_{L}\right]= & \frac{1}{2} \sum_{\mathbf{n}, \mathbf{m}} \varphi_{L}(\mathbf{n})\left(\square_{E, L}^{2 \alpha, \pm}+M_{L}^{2}\right) \varphi_{L}(\mathbf{m}) \\
& +\sum_{\mathbf{m}} \varphi_{L}(\mathbf{m}) J_{L}(\mathbf{m}) .
\end{aligned}
$$

For lattice theory with the lattice Riesz fractional Laplacian the action is

$$
\begin{aligned}
S_{E}\left[\varphi_{L}, J_{L}\right]= & \frac{1}{2} \sum_{\mathbf{n}, \mathbf{m}} \varphi_{L}(\mathbf{n})\left((-\Delta)_{L}^{\alpha / 2}+M_{L}^{2}\right) \varphi_{L}(\mathbf{m}) \\
& +\sum_{\mathbf{m}} \varphi_{L}(\mathbf{m}) J_{L}(\mathbf{m}) .
\end{aligned}
$$

Using (67), we rewrite expressions (75) in the form

$$
\begin{aligned}
S_{E}\left[\varphi_{L}, J_{L}\right]= & \frac{1}{2} \sum_{\mu=1 n_{\mu}, m_{\mu}=-\infty}^{+\infty} \varphi_{L}(\mathbf{n}) P_{n_{\mu} m_{\mu}}(2 \alpha) \varphi_{L}(\mathbf{m}) \\
& +\sum_{\mathbf{m}} \varphi_{L}(\mathbf{m}) J_{L}(\mathbf{m}),
\end{aligned}
$$

where the kernel $P_{n_{\mu} m_{\mu}}(2 \alpha)$ is given by

$$
\begin{aligned}
& P_{n_{\mu} m_{\mu}}(2 \alpha) \\
& =\frac{1}{a_{\mu}^{2 \alpha}} \frac{\pi^{2 \alpha}}{2 \alpha+1}{ }_{1} F_{2}\left(\frac{2 \alpha+1}{2} ; \frac{1}{2}, \frac{2 \alpha+3}{2} ;-\frac{\pi^{2}\left(n_{\mu}-m_{\mu}\right)^{2}}{4}\right) \\
& \quad+M_{L}^{2} \delta_{n_{\mu}, m_{\mu}},
\end{aligned}
$$

where ${ }_{1} F_{2}$ is the Gauss hypergeometric function $[33,34]$. Expression (78) can be used for all positive real values $\alpha$ including positive integer values. This kernel describes the space-time lattice with long-range properties that can be interpreted as a lattice space-time with power-law nonlocality. For the lattice with the nearest-neighbor interactions, the kernel $P_{n_{\mu} m_{\mu}}(\alpha)$ can defined by

$$
\begin{aligned}
P_{n_{\mu} m_{\mu}}(2)= & -\frac{1}{a_{\mu}^{2}} \sum_{s_{\mu}>0}\left(\delta_{n_{\mu}+s_{\mu}, m_{\mu}}+\delta_{n_{\mu}-s_{\mu}, m_{\mu}}-2 \delta_{n_{\mu}, m_{\mu}}\right) \\
& +M_{L}^{2} \delta_{n_{\mu}, m_{\mu}} .
\end{aligned}
$$

Note that the kernel (78) with $\alpha=2$ reproduces the same continuum fractional field theory as (79).

Using (68), we rewrite expression (76) in the form

$$
\begin{aligned}
S_{E}\left[\varphi_{L}, J_{L}\right]= & \frac{1}{2} \sum_{n, m=-\infty}^{+\infty} \varphi_{L}(\mathbf{n}) P_{\mathbf{n m}}(\alpha) \varphi_{L}(\mathbf{m}) \\
& +\sum_{\mathbf{m}} \varphi_{L}(\mathbf{m}) J_{L}(\mathbf{m}),
\end{aligned}
$$


where the kernel $P_{n_{\mu} m_{\mu}}(2 \alpha)$ is given by

$$
P_{\mathrm{nm}}(\alpha)=\frac{1}{a^{\alpha}} \mathscr{K}_{\alpha}^{+}(\mathbf{n}-\mathbf{m})+\sum_{\mu=1}^{4} M_{\mathrm{L}}^{2} \delta_{n_{\mu}, m_{\mu}}
$$

and $\mathscr{K}_{\alpha}^{+}(\mathbf{n}-\mathbf{m})$ is defined by the expression (69).

For the lattice fractional field theory we can define the generating functional in the form

$$
Z_{0, L}\left[J_{L}\right]=\int \prod_{\mathbf{n}} d \varphi_{L}(\mathbf{n}) e^{-S_{E}\left[\varphi_{L}, J_{L}\right]}
$$

It can be easily calculated, since the multiple integral is of the Gaussian type. Apart from an overall constant, which we will always drop since it plays no role when computing ensemble averages, we have that

$$
\begin{aligned}
& Z_{0, L}\left[J_{L}\right] \\
& =\frac{1}{\sqrt{\operatorname{det} P(2 \alpha)}} \exp \left(\frac{1}{2} \sum_{\mu=1 n_{\mu}, m_{\mu}=-\infty}^{+\infty} J_{L}(\mathbf{n}) P_{n_{\mu} m_{\mu}}^{-1}(2 \alpha) J_{L}(\mathbf{m})\right),
\end{aligned}
$$

where $P_{n_{\mu} m_{\mu}}^{-1}(2 \alpha)$ is the inverse of the matrix (78) and $\operatorname{det} P(2 \alpha)$ is the determinant of $P_{n_{\mu} m_{\mu}}^{-1}(2 \alpha)$. The inverse matrix $P_{n_{\mu} m_{\mu}}^{-1}(2 \alpha)$ is defined by the equation

$$
\sum_{s=-\infty}^{+\infty} P_{n_{\mu} s_{\mu}} P_{s_{\mu} m_{\mu}}^{-1}=\delta_{n_{\mu} m_{\mu}}, \quad(\mu=1,2,3,4)
$$

and it can be easily derived by using the momentum space, where $\delta_{n_{\mu} m_{\nu}}$ is given by

$$
\delta_{n_{\mu} m_{\mu}}=\frac{1}{k_{0 \mu}} \int_{-k_{0 \mu} / 2}^{+k_{0 \mu} / 2} d k_{\mu} e^{i k_{\mu}\left(n_{\mu}-m_{\mu}\right) a_{\mu}},
$$

where $k_{0 \mu} / 2=\pi / a_{\mu}$ and the integration is restricted by the Brillouin zone, $k_{\mu} \in\left[-k_{0 \mu} / 2, k_{0 \mu} / 2\right]$.

Using the discrete Fourier representation, one finds that $P_{n_{\mu} m_{\mu}}(2 \alpha)$ is given by

$$
\begin{aligned}
P_{n_{\mu} m_{\mu}}(2 \alpha) & =\mathscr{F}_{\Delta}^{-1}\left\{\widehat{P}_{2 \alpha}\left(k_{\mu}\right)\right\} \\
& =\frac{1}{k_{0 \mu}} \int_{-k_{0 \mu} / 2}^{+k_{0 \mu} / 2} d k_{\mu} \widehat{P}_{2 \alpha}\left(k_{\mu}\right) e^{i k_{\mu}\left(n_{\mu}-m_{\mu}\right) a_{\mu}},
\end{aligned}
$$

where

$$
\widehat{P}_{2 \alpha}\left(k_{\mu}\right)=\left|k_{\mu}\right|^{2 \alpha}+M_{L}^{2}
$$

Note that the integration in (86) is restricted to the Brillouin zone, $k_{\mu} \in\left[-k_{0 \mu} / 2, k_{0 \mu} / 2\right]$, where $\mu=1,2,3,4$ and $k_{0 \mu} / 2=$ $\pi / a_{\mu}$.

The inverse matrix is

$$
\begin{aligned}
P_{n_{\mu} m_{\mu}}^{-1}(2 \alpha) & =\mathscr{F}_{\Delta}^{-1}\left\{\widehat{P}_{2 \alpha}^{-1}\left(k_{\mu}\right)\right\} \\
& =\frac{1}{k_{0 \mu}} \int_{-k_{0 \mu} / 2}^{+k_{0 \mu} / 2} d k_{\mu} \frac{e^{i k_{\mu}\left(n_{\mu}-m_{\mu}\right) a_{\mu}}}{\left|k_{\mu}\right|^{2 \alpha}+M_{L}^{2}} .
\end{aligned}
$$

For the action (80) the generating functional is defined by the equation

$$
Z_{0, L}\left[J_{L}\right]=\frac{1}{\sqrt{\operatorname{det} P(\alpha)}} \exp \left(\frac{1}{2} \sum_{\mathbf{n}, \mathbf{m}} J_{L}(\mathbf{n}) P_{\mathbf{n m}}^{-1}(\alpha) J_{L}(\mathbf{m})\right) .
$$

Using the integer-order differentiation of (89) with respect to the sources $J_{L}$, we can obtain the correlation functions for the lattice fractional field theory. The 2-point correlation function is

$$
\left\langle\varphi_{L}(\mathbf{n}) \varphi_{L}(\mathbf{m})\right\rangle=\frac{\delta^{2} Z_{0, L}\left[J_{L}\right]}{\delta J_{L}(\mathbf{n}) \delta J_{L}(\mathbf{m})}=P_{\mathbf{n m}}^{-1}(\alpha) .
$$

Using the discrete Fourier representation, one finds that $P_{\mathrm{nm}}(\alpha)$ is given by

$$
\begin{aligned}
P_{\mathbf{n m}}(\alpha)= & \mathscr{F}_{\Delta}^{-1}\left\{\widehat{P}_{\alpha}(\mathbf{k})\right\} \\
= & \left(\prod_{\mu=1}^{4} \frac{1}{k_{0 \mu}}\right) \\
& \times \int_{-k_{01} / 2}^{+k_{01} / 2} \cdots \int_{-k_{04} / 2}^{+k_{04} / 2} d^{4} \mathbf{k} \widehat{P}_{\alpha}(\mathbf{k}) e^{i(\mathbf{k},(\mathbf{x}(\mathbf{n})-\mathbf{x}(\mathbf{m})))},
\end{aligned}
$$

where $k_{0 \mu}=2 \pi / a_{\mu}$ and

$$
\widehat{P}_{\alpha}(\mathbf{k})=|\mathbf{k}|^{\alpha}+M_{L}^{2}=\left(\sum_{\mu=1}^{4} k_{\mu}^{2}\right)^{\alpha / 2}+M_{L}^{2} .
$$

Here we use the notations

$$
\mathbf{x}(\mathbf{n})=\sum_{\mu=1}^{4} n_{\mu} \mathbf{a}_{\mu}, \quad(\mathbf{k}, \mathbf{x}(\mathbf{n}))=\sum_{\mu=1}^{4} k_{\mu} n_{\mu} a_{\mu} .
$$

The inverse matrix $P_{\mathrm{nm}}^{-1}(\alpha)$ has the form

$$
\begin{aligned}
P_{\mathbf{n m}}^{-1}(\alpha)= & \mathscr{F}_{\Delta}^{-1}\left\{\widehat{P}_{\alpha}^{-1}(\mathbf{k})\right\} \\
= & \left(\prod_{\mu=1}^{4} \frac{1}{k_{0 \mu}}\right) \\
& \times \int_{-k_{01} / 2}^{+k_{01} / 2} \cdots \int_{-k_{04} / 2}^{+k_{04} / 2} d^{4} \mathbf{k}\left(\widehat{P}_{\alpha}(\mathbf{k})\right)^{-1} e^{i(\mathbf{k},(\mathbf{x}(\mathbf{n})-\mathbf{x}(\mathbf{m})))}
\end{aligned}
$$

The right-hand side of expression (94) depends on the lattice sites $\mathbf{n}$ and $\mathbf{m}$ and on the dimensionless mass parameter $M_{L}$. Let us indicate this dependence explicitly, by using the notation $G_{P}\left(\mathbf{n}, \mathbf{m}, M_{L}, \alpha\right)=P_{\mathbf{n m}}^{-1}(\alpha)$. Then substituting (92) into (94), we have

$$
\begin{aligned}
G_{P}\left(\mathbf{n}, \mathbf{m}, M_{L}, \alpha\right)= & \left(\prod_{\mu=1}^{4} \frac{1}{k_{0 \mu}}\right) \\
& \times \int_{-k_{01} / 2}^{+k_{01} / 2} \cdots \int_{-k_{04} / 2}^{+k_{04} / 2} \frac{e^{i(\mathbf{k},(\mathbf{x}(\mathbf{n})-\mathbf{x}(\mathbf{m})))} d^{4} \mathbf{k}}{\left(\sum_{\mu=1}^{4} k_{\mu}^{2}\right)^{\alpha / 2}+M_{L}^{2}} .
\end{aligned}
$$


We can study continuum limit of (95) in order to extract the physical two-point correlation function, $\left\langle\varphi_{C}(\mathbf{x}) \varphi_{C}(\mathbf{y})\right\rangle$. To take the limit $a_{\mu} \rightarrow 0$, we should take into account that $x_{\mu} \rightarrow$ $n_{\mu} a_{\mu}$ and $y_{\mu} \rightarrow m_{\mu} a_{\mu}$. In our case, the continuum limit can give the correct continuum limit

$$
\left\langle\varphi_{\mathrm{C}}(\mathbf{x}) \varphi_{\mathrm{C}}(\mathbf{y})\right\rangle_{E}=\lim _{a_{\mu} \rightarrow 0} G_{P}\left(\sum_{\mu=1}^{4} \frac{x_{\mu}}{a_{\mu}} \mathbf{e}_{\mu}, \sum_{\mu=1}^{4} \frac{y_{\mu}}{a_{\mu}} \mathbf{e}_{\mu}, M_{\mathrm{C}}, \alpha\right)
$$

that reproduces the result for the scalar two-point function for fractional filed theory with continuum space-time.

\section{Continuum Fractional Field Theory from Lattice Theory}

In this section, we use the methods suggested in [18-20] to define the operation that transforms a lattice field $\varphi_{L}(\mathbf{n})$ and lattice operators into a field $\varphi_{C}(\mathbf{x})$ and operators for continuum space-time.

The transformation of the field is following. We consider the lattice scalar field $\varphi_{C}(\mathbf{n})$ as Fourier series coefficients of some function $\widehat{\varphi}(\mathbf{k})$ for $k_{\mu} \in\left[-k_{0 \mu} / 2, k_{0 \mu} / 2\right]$, where $\mu=$ $1,2,3,4$ and $k_{0 \mu} / 2=\pi / a_{\mu}$. As a next step we use the continuous limit $a_{\mu} \rightarrow 0+\left(\mathbf{k}_{0} \rightarrow \infty\right)$ to obtain $\widetilde{\varphi}(\mathbf{k})$. Finally we apply the inverse Fourier integral transform to obtain the continuum scalar field $\varphi_{C}(\mathbf{x})$. Let us give some details for these transformations of a lattice field into a continuum field [18-20].

The lattice-continuum transform operation $\mathscr{T}_{L \rightarrow C}$ is the combination of the operations $\mathscr{F}^{-1}$, Lim, and $\mathscr{F}_{\Delta}$ in the form

$$
\mathscr{T}_{L \rightarrow C}=\mathscr{F}^{-1} \circ \operatorname{Lim} \circ \mathscr{F}_{\Delta}
$$

that maps lattice field theory into the continuum field theory, where these operations are defined by the following.

(1) The Fourier series transform $\varphi_{L}(\mathbf{n}) \rightarrow \mathscr{F}_{\Delta}\left\{\varphi_{L}(\mathbf{n})\right\}=$ $\widehat{\varphi}(\mathbf{k})$ of the lattice scalar field $\varphi_{L}(\mathbf{n})$ is defined by

$$
\widehat{\varphi}(\mathbf{k})=\mathscr{F}_{\Delta}\left\{\varphi_{L}(\mathbf{n})\right\}=\sum_{n_{1}, \ldots, n_{4}=-\infty}^{+\infty} \varphi_{L}(\mathbf{n}) e^{-i(\mathbf{k}, \mathbf{x}(\mathbf{n}))},
$$

where the inverse Fourier series transform is

$$
\begin{aligned}
\varphi_{L}(\mathbf{n}) & =\mathscr{F}_{\Delta}^{-1}\{\widehat{\varphi}(\mathbf{k})\} \\
& =\left(\prod_{\mu=1}^{4} \frac{1}{k_{0 \mu}}\right) \int_{-k_{01} / 2}^{+k_{01} / 2} \cdots \int_{-k_{04} / 2}^{+k_{04} / 2} d^{4} \mathbf{k} \widehat{\varphi}(\mathbf{k}) e^{i(\mathbf{k}, \mathbf{x}(\mathbf{n}))} .
\end{aligned}
$$

Here we use the notations

$$
\mathbf{x}(\mathbf{n})=\sum_{\mu=1}^{4} n_{\mu} \mathbf{a}_{\mu}, \quad(\mathbf{k}, \mathbf{x}(\mathbf{n}))=\sum_{\mu=1}^{4} k_{\mu} n_{\mu} a_{\mu}
$$

and $a_{\mu}=2 \pi / k_{0 \mu}$ is the lattice constants.

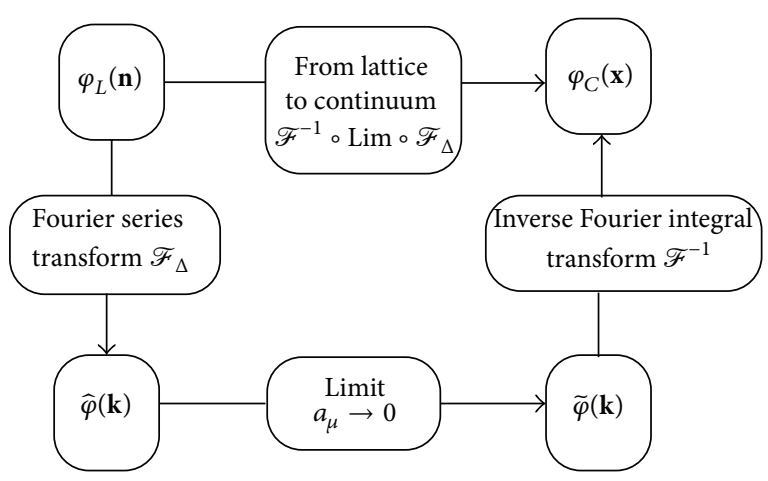

FIGURE 1: Diagram of sets of operations for scalar fields.

(2) The passage to the limit $\widehat{\varphi}(\mathbf{k}) \rightarrow \operatorname{Lim}\{\widehat{\varphi}(\mathbf{k})\}=\widetilde{\varphi}(\mathbf{k})$, where we use $a_{\mu} \rightarrow 0$ (or $k_{0 \mu} \rightarrow \infty$ ), allows us to derive the function $\widetilde{\varphi}(\mathbf{k})$ from $\widehat{\varphi}(\mathbf{k})$. By definition $\widetilde{\varphi}(\mathbf{k})$ is the Fourier integral transform of the continuum field $\varphi_{C}(\mathbf{x})$, and the function $\widehat{\varphi}(k)$ is the Fourier series transform of the lattice field $\varphi_{L}(\mathbf{n})$, where

$$
\varphi_{L}(\mathbf{n})=\left(\prod_{\mu=1}^{4} \frac{2 \pi}{k_{0 \mu}}\right) \varphi_{C}(\mathbf{x}(\mathbf{n}))
$$

and $\mathbf{x}(\mathbf{n})=n_{\mu} a_{\mu}=2 \pi n_{\mu} / k_{0 \mu} \rightarrow \mathbf{x}$. Note that $2 \pi / k_{0 \mu}=a_{\mu}$.

(3) The inverse Fourier integral transform $\widetilde{\varphi}(\mathbf{k}) \rightarrow$ $\mathscr{F}^{-1}\{\widetilde{\varphi}(\mathbf{k})\}=\varphi_{C}(\mathbf{x})$ is defined by

$$
\varphi_{C}(\mathbf{x})=\frac{1}{(2 \pi)^{4}} \int_{\mathbb{R}^{4}} d^{4} \mathbf{k} e^{i(\mathbf{k}, \mathbf{x})} \widetilde{\varphi}(\mathbf{k})=\mathscr{F}^{-1}\{\widetilde{\varphi}(\mathbf{k})\},
$$

where $(\mathbf{k}, \mathbf{x})=\sum_{\mu=1}^{4} k_{\mu} x_{\mu}$, and the Fourier integral transform of the continuum scalar field $\varphi_{C}(\mathbf{x})$ is

$$
\widetilde{\varphi}(\mathbf{k})=\int_{\mathbb{R}^{4}} d^{4} \mathbf{x} e^{-i(\mathbf{k}, \mathbf{x})} \varphi_{C}(\mathbf{x})=\mathscr{F}\left\{\varphi_{C}(\mathbf{x})\right\} .
$$

These transformations can be represented by the diagram in Figure 1.

Comparing (98)-(99) and (102)-(103), we see the existence of a cut-off in the momentum in the lattice field theory. In the theory of the lattice fields $\varphi_{L}(\mathbf{n})$, the momentum integration with respect to the wave-vector components $k_{\mu}$ is restricted by the Brillouin zones $k \in\left[-k_{0 \mu} / 2, k_{0 \mu} / 2\right]$, where $k_{0 \mu}=$ $2 \pi / a_{\mu}$.

In the lattice 4-dimensional space-time, all four components of momenta $k_{\mu}$ are restricted by the interval $k \in$ $\left[-k_{0 \mu} / 2, k_{0 \mu} / 2\right]$. Therefore the introduction of a lattice spacetime provides a momentum cut-off of the order of the inverse lattice constants, $k_{0 \mu}=2 \pi / a_{\mu}$.

Using the lattice-continuum transform operation $\mathscr{T}_{L \rightarrow C}$, (95) and (96) give the expression for the continuum fractional field theory:

$$
\left\langle\varphi_{C}(\mathbf{x}) \varphi_{C}(\mathbf{y})\right\rangle_{E}=\frac{1}{(2 \pi)^{4}} \int_{\mathbb{R}^{4}} d^{4} \mathbf{k} \frac{e^{i(\mathbf{k}, \mathbf{x}-\mathbf{y})}}{\left(\sum_{\mu=1}^{4} k_{\mu}^{2}\right)^{\alpha / 2}+M_{C}^{2}} .
$$


Let us formulate and prove a proposition about the connection between the lattice fractional derivative and continuum fractional derivatives of noninteger orders with respect to coordinates.

Proposition 8. The lattice-continuum transform operation $\mathscr{T}_{L \rightarrow C}$ maps the lattice fractional derivatives

$$
\left(\mathbb{D}_{L}^{ \pm}\left[\begin{array}{l}
\alpha \\
\mu
\end{array}\right] \varphi_{L}\right)(\mathbf{n})=\frac{1}{a_{\mu}^{\alpha}} \sum_{m_{\mu}=-\infty}^{+\infty} K_{\alpha}^{ \pm}\left(n_{\mu}-m_{\mu}\right) \varphi_{L}(\mathbf{m}),
$$

where $K_{\alpha}^{ \pm}(n-m)$ are defined by (47), (48), into the continuum fractional derivatives of order $\alpha$ with respect to coordinate $x_{\mu}$ by

$$
\mathscr{T}_{L \rightarrow C}\left(\mathbb{D}_{L}^{ \pm}\left[\begin{array}{l}
\alpha \\
\mu
\end{array}\right] \varphi_{L}(\mathbf{m})\right)=\mathbb{D}_{C}^{ \pm}\left[\begin{array}{l}
\alpha \\
\mu
\end{array}\right] \varphi_{C}(\mathbf{x}) .
$$

Proof. Let us multiply (105) by the expression $\exp \left(-i k_{\mu} n_{\mu} a_{\mu}\right)$ and then sum over $n_{\mu}$ from $-\infty$ to $+\infty$. Then

$$
\begin{aligned}
\mathscr{F}_{\Delta}\left(\mathbb{D}_{L}^{ \pm}\left[\begin{array}{l}
\alpha \\
\mu
\end{array}\right] \varphi_{L}(\mathbf{m})\right) \\
=\sum_{n_{\mu}=-\infty}^{+\infty} e^{-i k_{\mu} n_{\mu} a_{\mu}} \mathbb{D}_{L}^{ \pm}\left[\begin{array}{l}
\alpha \\
\mu
\end{array}\right] \varphi_{L}(\mathbf{m}) \\
=\frac{1}{a_{\mu}} \sum_{n_{\mu}=-\infty}^{+\infty} \sum_{m_{\mu}=-\infty}^{+\infty} e^{-i k_{\mu} n_{\mu} a_{\mu}} K_{\alpha}^{ \pm}\left(n_{\mu}-m_{\mu}\right) \varphi_{L}(\mathbf{m}) .
\end{aligned}
$$

Using (98), the right-hand side of (107) gives

$$
\begin{aligned}
& \sum_{n_{\mu}=-\infty}^{+\infty} \sum_{m_{\mu}=-\infty}^{+\infty} e^{-i k_{\mu} n_{\mu} a_{\mu}} K_{\alpha}^{ \pm}\left(n_{\mu}-m_{\mu}\right) \varphi_{L}(\mathbf{m}) \\
& =\sum_{n_{\mu}=-\infty}^{+\infty} e^{-i k_{\mu} n_{\mu} a_{\mu}} K_{\alpha}^{ \pm}\left(n_{\mu}-m_{\mu}\right) \sum_{m_{\mu}=-\infty}^{+\infty} \varphi_{L}(\mathbf{m}) \\
& =\sum_{n_{\mu}^{\prime}=-\infty}^{+\infty} e^{-i k_{\mu} n_{\mu}^{\prime} a_{\mu}} K_{\alpha}^{ \pm}\left(n_{\mu}^{\prime}\right) \\
& \quad \times \sum_{m_{\mu}=-\infty}^{+\infty} \varphi_{L}(\mathbf{m}) e^{-i k_{\mu} m_{\mu} a_{\mu}}=\widehat{K}_{\alpha}^{ \pm}\left(k_{\mu} a_{\mu}\right) \widehat{\varphi}(\mathbf{k}),
\end{aligned}
$$

where $n_{\mu}^{\prime}=n_{\mu}-m_{\mu}$.

As a result, (107) has the form

$$
\mathscr{F}_{\Delta}\left(\mathbb{D}_{L}^{ \pm}\left[\begin{array}{l}
\alpha \\
\mu
\end{array}\right] \varphi_{L}(\mathbf{m})\right)=\frac{1}{a_{\mu}^{\alpha}} \widehat{K}_{\alpha}^{ \pm}\left(k_{\mu} a_{\mu}\right) \widehat{\varphi}(\mathbf{k}),
$$

where $\mathscr{F}_{\Delta}$ is an operator notation for the discrete Fourier transform.

Then we use

$$
\begin{gathered}
\widehat{K}_{\alpha}^{+}\left(a_{\mu} k_{\mu}\right)=\left|a_{\mu} k_{\mu}\right|^{\alpha}, \\
\widehat{K}_{\alpha}^{-}\left(a_{\mu} k_{\mu}\right)=i \operatorname{sgn}\left(k_{\mu}\right)\left|a_{\mu} k_{\mu}\right|^{\alpha},
\end{gathered}
$$

and the limit $a_{\mu} \rightarrow 0$ gives

$$
\begin{gathered}
\widetilde{K}_{\alpha}^{+}\left(k_{\mu}\right)=\lim _{a_{\mu} \rightarrow 0} \frac{1}{a_{\mu}^{\alpha}} \widehat{K}_{\alpha}^{+}\left(k_{\mu} a_{\mu}\right)=\left|k_{\mu}\right|^{\alpha}, \\
\widetilde{K}_{\alpha}^{-}\left(k_{\mu}\right)=\lim _{a_{\mu} \rightarrow 0} \frac{1}{a_{\mu}^{\alpha}} \widehat{K}_{\alpha}^{-}\left(k_{\mu} a_{\mu}\right)=i k_{\mu}\left|k_{\mu}\right|^{\alpha-1} .
\end{gathered}
$$

As a result, the limit $a_{\mu} \rightarrow 0$ for (109) gives

$$
\operatorname{Lim} \circ \mathscr{F}_{\Delta}\left(\mathbb{D}_{L}^{ \pm}\left[\begin{array}{l}
\alpha \\
\mu
\end{array}\right] \varphi_{L}(\mathbf{m})\right)=\widehat{K}_{\alpha}^{ \pm}\left(k_{\mu}\right) \widehat{\varphi}(\mathbf{k}),
$$

where

$$
\begin{gathered}
\widetilde{K}_{\alpha}^{+}\left(k_{\mu}\right)=\left|k_{\mu}\right|^{\alpha}, \quad \widetilde{K}_{\alpha}^{-}\left(k_{\mu}\right)=i k_{\mu}\left|k_{\mu}\right|^{\alpha-1}, \\
\widetilde{\varphi}(\mathbf{k})=\operatorname{Lim} \widehat{\varphi}(\mathbf{k}) .
\end{gathered}
$$

The inverse Fourier transforms of (112) have the form

$$
\begin{array}{r}
\mathscr{F}^{-1} \circ \operatorname{Lim} \circ \mathscr{F}_{\Delta}\left(\mathbb{D}_{L}^{+}\left[\begin{array}{l}
\alpha \\
\mu
\end{array}\right] \varphi_{L}(\mathbf{m})\right)=\mathbb{D}_{C}^{+}\left[\begin{array}{l}
\alpha \\
\mu
\end{array}\right] \varphi_{C}(\mathbf{x}) \\
(\alpha>0), \\
\mathscr{F}^{-1} \circ \operatorname{Lim} \circ \mathscr{F}_{\Delta}\left(\mathbb{D}_{L}^{-}\left[\begin{array}{l}
\alpha \\
\mu
\end{array}\right] \varphi_{L}(\mathbf{m})\right)=\mathbb{D}_{C}^{-}\left[\begin{array}{l}
\alpha \\
\mu
\end{array}\right] \varphi_{C}(\mathbf{x}) \\
(\alpha>0),
\end{array}
$$

where we use the connection between the continuum fractional derivatives of the order $\alpha$ and the correspondent Fourier integrals transforms

$$
\begin{gathered}
\mathscr{F}\left(\mathbb{D}_{C}^{+}\left[\begin{array}{l}
\alpha \\
\mu
\end{array}\right] \varphi_{C}(\mathbf{x})\right)=\left|k_{\mu}\right|^{\alpha} \hat{\varphi}(\mathbf{k}), \\
\mathscr{F}\left(\mathbb{D}_{C}^{-}\left[\begin{array}{l}
\alpha \\
\mu
\end{array}\right] \varphi_{C}(\mathbf{x})\right)=i \operatorname{sgn}\left(k_{\mu}\right)\left|k_{\mu}\right|^{\alpha} \hat{\varphi}(\mathbf{k}) .
\end{gathered}
$$

As a result, we obtain that lattice fractional derivatives are transformed by the lattice-continuum transform operation $\mathscr{T}_{L \rightarrow C}$ into continuum fractional derivatives of the Riesz type.

This ends the proof.

We have similar relations for other lattice fractional differential operators. Using this Proposition, it is easy to prove that the lattice-continuum transform operation $\mathscr{T}_{L \rightarrow C}$ maps the lattice Laplace operators (65), (66), and (68) into the continuum 4-dimensional Laplacians of noninteger orders that are defined by (30), (31), and (35) such that we have

$$
\begin{aligned}
\mathscr{T}_{L \rightarrow C}\left(\left(\square_{E, L}^{2 \alpha, \pm} \varphi_{L}\right)(\mathbf{n})\right) & =\left(\square_{E, C}^{2 \alpha, \pm} \varphi_{C}\right)(\mathbf{x}), \\
\mathscr{T}_{L \rightarrow C}\left(\left(\square_{E, L}^{\alpha, \alpha, \pm} \varphi_{L}\right)(\mathbf{n})\right) & =\left(\square_{E, C}^{\alpha, \alpha, \pm} \varphi_{C}\right)(\mathbf{x}), \\
\mathscr{T}_{L \rightarrow C}\left(\left((-\Delta)_{L}^{\alpha / 2} \varphi_{L}\right)(\mathbf{n})\right) & =\left((-\Delta)_{C}^{\alpha / 2} \varphi_{C}\right)(\mathbf{x}) .
\end{aligned}
$$

As a result, the continuous limits of the lattice fractional field equations give the continuum fractional-order field equations for continuum space-time. 


\section{Conclusion}

In this paper, an approach to formulate the fractional field theory on a lattice space-time has been suggested. Note that lattice approaches to the fractional field theories were not previously considered. A fractional-order generalization of the lattice field theories has not been proposed before. The suggested approach, which is suggested in this paper, can be considered from two following points of view. Firstly it allows us to give lattice analogs of the fractional field theories. Secondly, it allows us to formulate fractional-order analogs of the lattice quantum field theories. The lattice analogs of the fractional-order derivatives for fields on the lattice space-time are suggested to formulate lattice fractional field theories. The space-time lattices are characterized by the long-range properties of power-law type instead of the usual lattices characterized by a nearest-neighbors presentation (or by a finite neighbor environment) usually used in lattice field theories. We prove that continuum limit of the lattice fractional theory gives the theory of fractional field on continuum space-time. The fractional field equations, which are obtained by continuum limit, contain the Riesz type derivatives on noninteger orders with respect to space-time coordinates.

\section{Conflict of Interests}

The author declares that there is no conflict of interests regarding the publication of this paper.

\section{References}

[1] S. G. Samko, A. A. Kilbas, and O. I. Marichev, Fractional Integ rals and Derivatives Theory and Applications, Gordon and Breach Science, New York, NY, USA, 1993.

[2] A. A. Kilbas, H. M. Srivastava, and J. J. Trujillo, Theory and Applications of Fractional Differential Equations, Elsevier, Amsterdam, The Netherlands, 2006.

[3] N. Laskin, "Fractional quantum mechanics and Lévy path integrals," Physics Letters A, vol. 268, no. 4-6, pp. 298-305, 2000.

[4] N. Laskin, "Fractional quantum mechanics," Physical Review E, vol. 62 , no. 3, pp. 3135-3145, 2000.

[5] V. E. Tarasov, "Weyl quantization of fractional derivatives," Journal of Mathematical Physics, vol. 49, no. 10, Article ID 102112, 6 pages, 2008.

[6] V. E. Tarasov, "Fractional Heisenberg equation," Physics Letters A, vol. 372, no. 17, pp. 2984-2988, 2008.

[7] V. E. Tarasov, "Fractional generalization of the quantum Markovian master equation," Theoretical and Mathematical Physics, vol. 158, no. 2, pp. 179-195, 2009.

[8] V. E. Tarasov, "Fractional dynamics of open quantum systems," in Fractional Dynamics. Recent Advances, J. Klafter, S. C. Lim, and R. Metzler, Eds., pp. 449-482, World Scientific, Singapore, 2011.

[9] V. E. Tarasov, Quantum Mechanics of Non-Hamiltonian and Dissipative Systems, Elsevier Science, 2008.

[10] G. Calcagni, "Quantum field theory, gravity and cosmology in a fractal universe," Journal of High Energy Physics, vol. 2010, article 120, 38 pages, 2010.

[11] G. Calcagni, "Geometry and field theory in multi-fractional spacetime," Journal of High Energy Physics, vol. 2012, article 65, 2012.
[12] S. C. Lim, "Fractional derivative quantum fields at positive temperature," Physica A, vol. 363, no. 2, pp. 269-281, 2006.

[13] S. C. Lim and L. P. Teo, "Casimir effect associated with fractional Klein-Gordon field," in Fractional Dynamics, J. Klafter, S. C. Lim, and R. Metzler, Eds., pp. 483-506, World Science Publisher, Singapore, 2012.

[14] M. Riesz, "L'intégrale de Riemann-Liouville et le problème de Cauchy," Acta Mathematica, vol. 81, no. 1, pp. 1-222, 1949 (French).

[15] C. G. Bollini and J. J. Giambiagi, "Arbitrary powers of d’Alembertians and the Huygens principle," Journal of Mathematical Physics, vol. 34, no. 2, pp. 610-621, 1993.

[16] D. G. Barci, C. G. Bollini, L. E. Oxman, and M. Rocca, "Lorentzinvariant pseudo-differential wave equations," International Journal of Theoretical Physics, vol. 37, no. 12, pp. 3015-3030, 1998.

[17] R. L. P. G. do Amaral and E. C. Marino, "Canonical quantization of theories containing fractional powers of the d'Alembertian operator," Journal of Physics A: Mathematical and General, vol. 25, no. 19, pp. 5183-5200, 1992.

[18] V. E. Tarasov, "Continuous limit of discrete systems with longrange interaction," Journal of Physics A: Mathematical and General, vol. 39, no. 48, pp. 14895-14910, 2006.

[19] V. E. Tarasov, "Map of discrete system into continuous," Journal of Mathematical Physics, vol. 47, no. 9, Article ID 092901, 24 pages, 2006.

[20] V. E. Tarasov, "Toward lattice fractional vector calculus," Journal of Physics A, vol. 47, no. 35, Article ID 355204, 2014.

[21] V. E. Tarasov, "Lattice model with power-law spatial dispersion for fractional elasticity," Central European Journal of Physics, vol. 11, no. 11, pp. 1580-1588, 2013.

[22] V. E. Tarasov, "Fractional gradient elasticity from spatial dispersion law," ISRN Condensed Matter Physics, vol. 2014, Article ID 794097, 13 pages, 2014.

[23] V. E. Tarasov, "Lattice with long-range interaction of power-law type for fractional non-local elasticity," International Journal of Solids and Structures, vol. 51, no. 15-16, pp. 2900-2907, 2014.

[24] V. E. Tarasov, "Lattice model of fractional gradient and integral elasticity: long-range interaction of Grünwald-Letnikov-Riesz type," Mechanics of Materials, vol. 70, no. 1, pp. 106-114, 2014.

[25] V. E. Tarasov, "Large lattice fractional Fokker-Planck equation," Journal of Statistical Mechanics: Theory and Experiment, vol. 2014, Article ID P09036, 2014.

[26] V. E. Tarasov, "Non-linear fractional field equations: weak nonlinearity at power-law non-locality," Nonlinear Dynamics, 2014.

[27] J. C. Collins, Renormalization: An Intro duction to Renormalization, the Renormaliza tion Group and the Operator-Product Expansion, Cambridge University Press, Cambridge, UK, 1984.

[28] M. Chaichian and A. Demichev, Path Integrals in Physics: Volume II Quantum Field Theory, Statistical Physics and other Modern Applications, Institute of Physics Publishing, Philadelphia, Pa, USA, CRC Press, 2001.

[29] K. Huang, Quarks, Leptons and Gauge Fields, World Scientific, Singapore, 2nd edition, 1992.

[30] V. V. Uchaikin, Fractional Derivatives for Physicists and Engineers. Volume I. Background and Theory, Nonlinear Physical Science, Springer, Berlin, Germany, Higher Education Press, Beijing, China, 2012.

[31] V. E. Tarasov, "No violation of the Leibniz rule. No fractional derivative," Communications in Nonlinear Science and Numerical Simulation, vol. 18, no. 11, pp. 2945-2948, 2013. 
[32] N. Laskin, "Fractional Schrödinger equation," Physical Review E: Statistical, Nonlinear, and Soft Matter Physics, vol. 66, no. 5, Article ID 056108, 7 pages, 2002.

[33] A. Erdelyi, W. Magnus, F. Oberhettinger, and F. G. Tricomi, Higher Transcendental Functions, vol. 1, McGraw-Hill, New York, NY, USA, 1953.

[34] A. Erdelyi, W. Magnus, F. Oberhettinger, and F. G. Tricomi, Higher Transcendental Functions, vol. 1, Krieeger, Melbourne, Australia, 1981.

[35] A. P. Prudnikov, Y. A. Brychkov, and O. I. Marichev, Integrals and Series. Volume 1. Elementary Functions, Gordon \& Breach Science Publishers, New York, NY, USA, 1986.

[36] V. E. Tarasov, Fractional Dynamics: Applications of Fractional Calculus to Dynamics of Particles, Fields and Media, Springer, New York, NY, USA, 2011. 

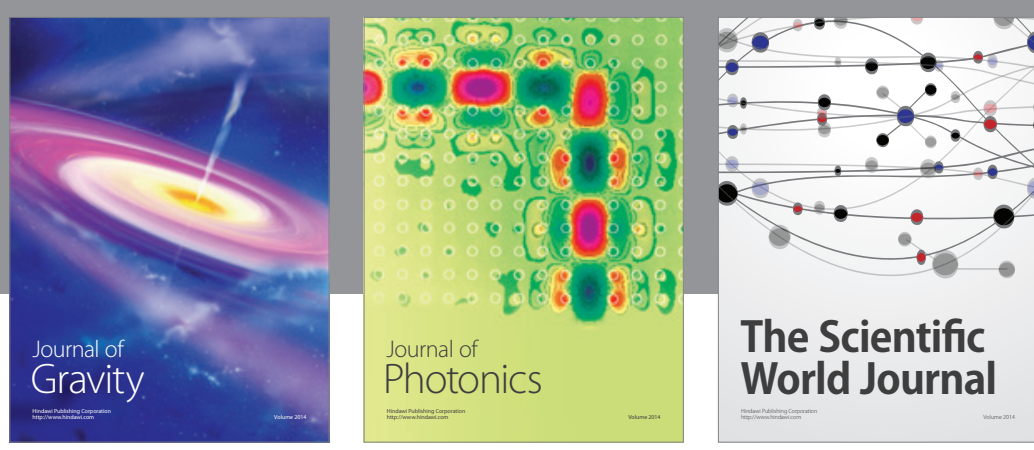

The Scientific World Journal
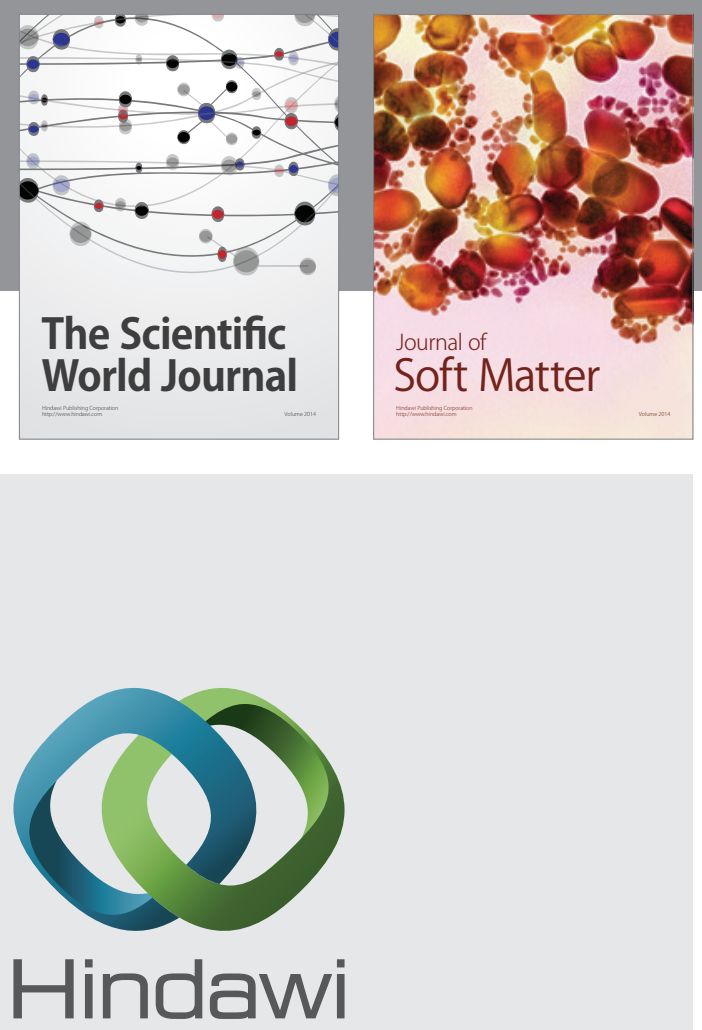

Submit your manuscripts at

http://www.hindawi.com

nternational Journal of

Statistical Mechanics
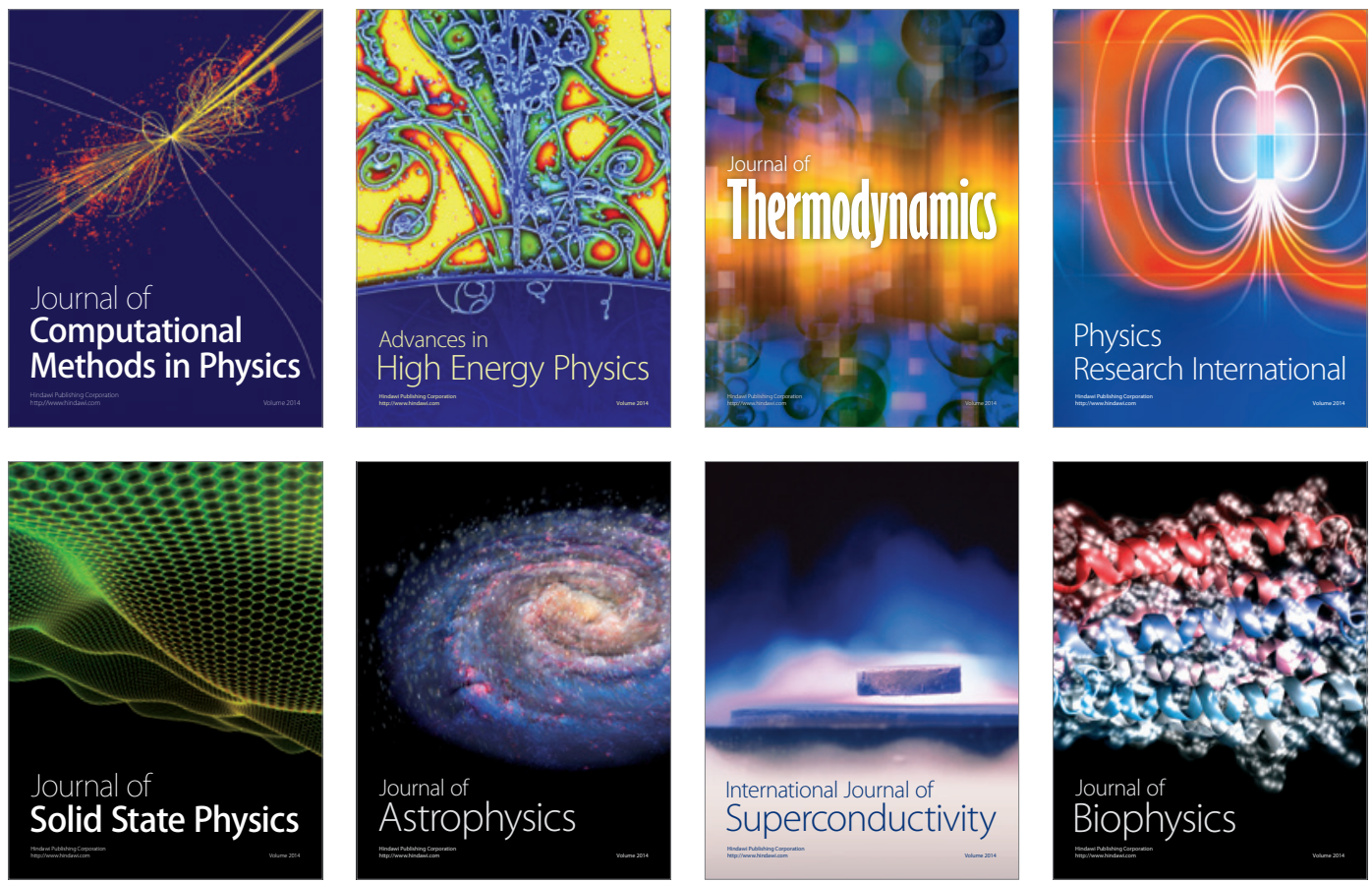
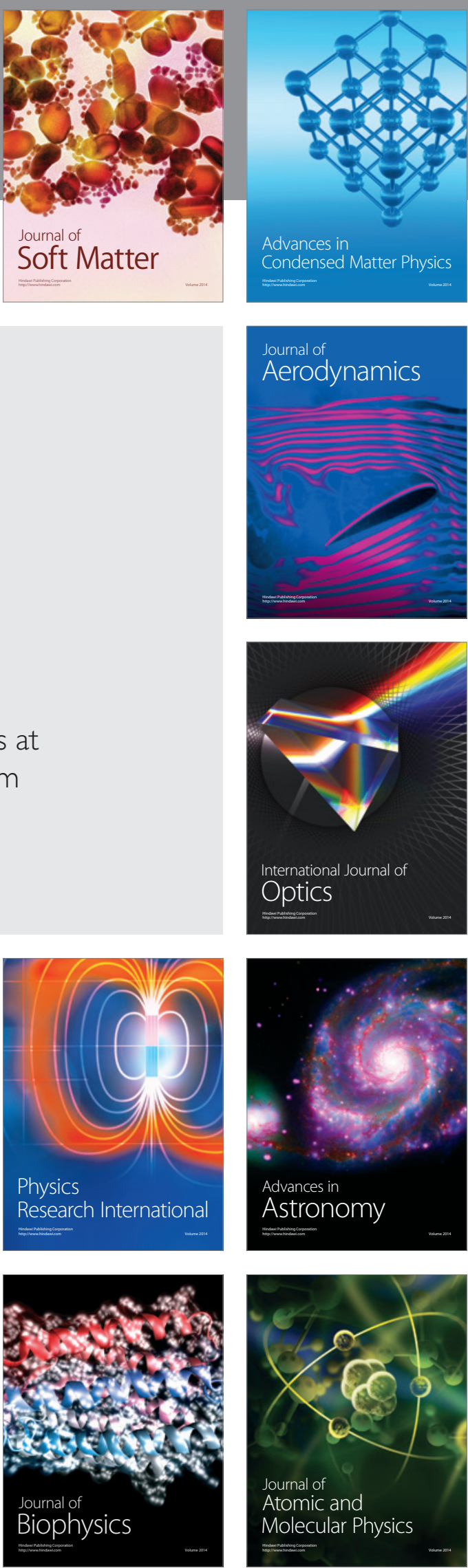